\title{
GENERALIZED FERMIONIC DISCRETE TODA HIERARCHY
}

\author{
V. V. GRIBANOV, V. G. KADYSHEVSKY, AND A. S. SORIN
}

Received 16 November 2003

To the memory of Professor I. Prigogine

We describe bi-Hamiltonian structure and Lax-pair formulation with the spectral parameter of the generalized fermionic Toda lattice hierarchy as well as its bosonic and fermionic symmetries for different (including periodic) boundary conditions. Its two reductions $-N=4$ and $N=2$ supersymmetric Toda lattice hierarchies-in different (including canonical) bases are investigated. Its $r$-matrix description, monodromy matrix, and spectral curves are discussed.

\section{Introduction}

At present, two different nontrivial supersymmetric extensions of the two-dimensional (2D) infinite bosonic Toda lattice (TL) hierarchy are known. They are the $N=(2 \mid 2)$ $[6,8,9,11,15,16]$ and $N=(0 \mid 2)[11]$ supersymmetric TL hierarchies. Actually, besides a different number of supersymmetries, they have different bosonic limits which are decoupled systems of two infinite bosonic TL hierarchies and single infinite bosonic TL hierarchy, respectively. One-dimensional (1D) reductions of these hierarchies $-N=4$ and $N=2$ supersymmetric TL hierarchies-were studied in [3, 4], while their finite reductions corresponding to different boundary conditions (e.g., fixed ends, periodic boundary conditions, etc.) were investigated in $[1,2,5,17,18]$. Quite recently, a dispersionless limit of the $N=(1 \mid 1)$ supersymmetric TL hierarchy was constructed in $[10,12]$.

The present paper continues studies of the above-mentioned hierarchies and is addressed to yet unsolved problems of constructing their periodic counterparts, biHamiltonian structure in different (including canonical) bases, $2 m \times 2 m$ matrix and $4 \times 4$ matrix $(3 \times 3$ matrix) Lax-pair descriptions with the spectral parameter, $r$-matrix approach, and spectral curves.

The structure of this paper is as follows. In Section 2.1, starting with the zero-curvature representation, we introduce the $2 \mathrm{D}$ generalized fermionic TL equations and describe their two reductions related to the $N=(2 \mid 2)$ and $N=(0 \mid 2)$ supersymmetric TL equations. Then, in Section 2.2, we construct the bi-Hamiltonian structure of the 1D generalized fermionic TL hierarchy and its fermionic and bosonic Hamiltonians. 
Sections 3 and 4 are devoted to the $1 \mathrm{D} N=4$ and $N=2$ supersymmetric TL hierarchies, respectively. We construct their bi-Hamiltonian structure in Sections 3.1 and 4.1, fermionic symmetries in Section 3.2, and in Sections 3.3 and 4.2, we investigate a transition to the canonical basis which spoils a number of supersymmetries.

In Section 5, we consider periodic supersymmetric TL hierarchies. Thus, in Section 5.1, we construct the $2 m \times 2 m$ matrix zero-curvature representation with the spectral parameter for the periodic 2D generalized fermionic TL hierarchy. Then, in Section 5.2, we obtain the bi-Hamiltonian structure of its $1 \mathrm{D}$ reduction. In Section 5.3, we construct the $4 \times 4$ matrix Lax-pair representation of this hierarchy, calculate its $r$-matrix, and analyze monodromy matrix. We next calculate its spectral curves in Section 5.4. In Section 5.5, we give a short summary of the $3 \times 3$ matrix Lax-pair representation and the $r$-matrix formalism for the periodic 1D $N=2$ TL hierarchy, and calculate spectral curves of the latter. In Section 5.6, we discuss periodic TL equations in the canonical basis and their fermionic symmetries.

\section{Generalized fermionic TL hierarchy}

2.1. 2D generalized fermionic TL equations. In this subsection, we define two-dimensional generalized fermionic TL equations and describe their two different representations which being reduced relate them with the $N=(2 \mid 2)[5,6]$ and $N=(0 \mid 2)[5,11]$ supersymmetric TL equations.

Our starting point is the following zero-curvature representation:

$$
\left[\partial_{1}+L^{-}, \partial_{2}-L^{+}\right]=0
$$

for the infinite matrices

$$
\begin{aligned}
\left(L^{-}\right)_{i, j}= & \rho_{i} \delta_{i, j+1}+d_{i} \delta_{i, j+2}, \\
L^{-} & =\left(\begin{array}{cccccccc}
\cdots & & \left(L^{+}\right)_{i, j}=\delta_{i, j-2}+\gamma_{i} \delta_{i, j-1}+c_{i} \delta_{i, j}, \\
\cdots & 0 & 0 & 0 & 0 & 0 & 0 & \cdots \\
& \rho_{j+1} & 0 & 0 & 0 & 0 & 0 & \\
& d_{j+2} & \rho_{j+2} & 0 & 0 & 0 & 0 & \\
& 0 & d_{j+3} & \rho_{j+3} & 0 & 0 & 0 & \\
& 0 & 0 & d_{j+4} & \rho_{j+4} & 0 & 0 & \\
\cdots & 0 & 0 & 0 & d_{j+5} & \rho_{j+5} & 0 & \cdots \\
\cdots & \cdots & & & & & \cdots & \cdots
\end{array}\right), \\
L^{+} & =\left(\begin{array}{cccccccc}
\cdots & \cdots & & & & & \cdots & \cdots \\
\cdots & c_{j} & \gamma_{j} & 1 & 0 & 0 & 0 & \cdots \\
& 0 & c_{j+1} & \gamma_{j+1} & 1 & 0 & 0 & \\
0 & 0 & c_{j+2} & \gamma_{j+2} & 1 & 0 & \\
& 0 & 0 & 0 & c_{j+3} & \gamma_{j+3} & 1 & \\
\cdots & 0 & 0 & 0 & 0 & c_{j+4} & \gamma_{j+4} & \\
\cdots & 0 & 0 & 0 & 0 & 0 & c_{j+5} & \cdots \\
\cdots & & & & & \cdots & \cdots
\end{array}\right) .
\end{aligned}
$$


Here, $z_{1}$ and $z_{2}$ are the bosonic coordinates $\left(\partial_{1,2} \equiv \partial / \partial z_{1,2}\right)$; the matrix entries $d_{j}, c_{j}$ $\left(\rho_{j}, \gamma_{j}\right)$ are the bosonic (fermionic) fields with Grassmann parity 0 (1) and length dimensions $\left[d_{j}\right]=-2,\left[c_{j}\right]=-1,\left[\rho_{j}\right]=-3 / 2$, and $\left[\gamma_{j}\right]=-1 / 2$. The zero-curvature representation (2.1) leads to the following system of evolution equations with respect to the bosonic evolution derivatives $\partial_{1,2}$ :

$$
\begin{aligned}
& \partial_{2} d_{j}=d_{j}\left(c_{j}-c_{j-2}\right), \quad \partial_{1} c_{j}=d_{j+2}-d_{j}+\gamma_{j} \rho_{j+1}+\gamma_{j-1} \rho_{j}, \\
& \partial_{1} \gamma_{j}=\rho_{j+2}-\rho_{j}, \quad \partial_{2} \rho_{j}=\rho_{j}\left(c_{j}-c_{j-1}\right)+d_{j+1} \gamma_{j}-d_{j} \gamma_{j-2} .
\end{aligned}
$$

Keeping in mind that in the bosonic limit (i.e., when all fermionic fields are put equal to zero) these equations describe a system of two decoupled bosonic 2D TLs, we call (2.3) the $2 \mathrm{D}$ generalized fermionic TL equations.

Our next goal is to describe fermionic symmetries of the 2D generalized fermionic TL equations (2.3). Before doing so, we first supply the fields $\left(d_{j}, c_{j}, \gamma_{j}, \rho_{j}\right)$ with boundary conditions. In what follows we consider the boundary conditions of the following four types:

$$
\begin{gathered}
\lim _{j \rightarrow \pm \infty} d_{j}=0, \quad \lim _{j \rightarrow \pm \infty} c_{j}=0, \quad \lim _{j \rightarrow \pm \infty} \gamma_{j}=0, \quad \lim _{j \rightarrow \pm \infty} \rho_{j}=0 ; \\
\lim _{j \rightarrow \pm \infty} d_{j}=1, \quad \lim _{j \rightarrow \pm \infty} c_{j}=0, \quad \lim _{j \rightarrow \pm \infty} \gamma_{j}=0, \quad \lim _{j \rightarrow \pm \infty} \rho_{j}=0 ; \\
\lim _{j \rightarrow \pm \infty} d_{2 j+1}=1, \quad \lim _{j \rightarrow \pm \infty} d_{2 j}=0, \quad \lim _{j \rightarrow \pm \infty} c_{j}=0, \\
\lim _{j \rightarrow \pm \infty} \gamma_{j}=0, \quad \lim _{j \rightarrow \pm \infty} \rho_{j}=0 ; \\
d_{j}=d_{j+n}, \quad c_{j}=c_{j+n}, \quad \gamma_{j}=\gamma_{j+n}, \quad \rho_{j}=\rho_{j+n}, \quad n \in \mathbb{Z} .
\end{gathered}
$$

The first three types specify the behavior of the fields at the lattice points at infinity, while the boundary condition of the fourth type is periodic and corresponds to the closed $2 \mathrm{D}$ generalized fermionic TL.

For the boundary conditions (2.4a) and (2.4b), the above-described (2.3) possess the $N=(2 \mid 2)$ supersymmetry. Indeed, in this case, there exist four fermionic symmetries of $(2.3)$ :

$$
\begin{array}{rlrl}
D_{1}^{1} d_{j} & =g_{j-1} \rho_{j}+g_{j} \rho_{j-1}, & D_{2}^{1} d_{j} & =(-1)^{j}\left(g_{j-1} \rho_{j}-g_{j} \rho_{j-1}\right), \\
D_{1}^{1} c_{j} & =g_{j} \gamma_{j-1}+g_{j+1} \gamma_{j}, & D_{2}^{1} c_{j}=(-1)^{j}\left(g_{j+1} \gamma_{j}-g_{j} \gamma_{j-1}\right), \\
D_{1}^{1} \rho_{j} & =-\partial_{1} g_{j}, & D_{2}^{1} \rho_{j} & =(-1)^{j} \partial_{1} g_{j}, \\
D_{1}^{1} \gamma_{j} & =g_{j}-g_{j+2}, & D_{2}^{1} \gamma_{j} & =(-1)^{j}\left(g_{j+2}-g_{j}\right), \\
D_{3}^{2} d_{j} & =d_{j}\left(\gamma_{j-1}+\gamma_{j-2}\right), & D_{4}^{2} d_{j}=(-1)^{j} d_{j}\left(\gamma_{j-1}-\gamma_{j-2}\right), \\
D_{3}^{2} c_{j} & =\partial_{2} \sum_{k=-\infty}^{j-1} \gamma_{k}, & D_{4}^{2} c_{j} & =-\partial_{2} \sum_{k=-\infty}^{j-1}(-1)^{k} \gamma_{k}, \\
D_{3}^{2} \rho_{j} & =d_{j+1}-d_{j}-\rho_{j} \gamma_{j-1},, & D_{4}^{2} \rho_{j} & =(-1)^{j}\left(d_{j+1}-d_{j}-\rho_{j} \gamma_{j-1}\right), \\
D_{3}^{2} \gamma_{j} & =c_{j+1}-c_{j}, & D_{4}^{2} \gamma_{j} & =(-1)^{j}\left(c_{j+1}-c_{j}\right),
\end{array}
$$


where $D_{1}^{1}, D_{2}^{1}, D_{3}^{2}$, and $D_{4}^{2}$ are the fermionic evolution derivatives; $g_{j}$ denotes the infinite product

$$
g_{j} \equiv \prod_{k=0}^{\infty} \frac{d_{j-2 k}}{d_{j-2 k-1}}
$$

with the properties $g_{j} g_{j-1}=d_{j}$ and

$$
\begin{gathered}
D_{1}^{1} g_{j}=\rho_{j}, \quad D_{2}^{1} g_{j}=(-1)^{j} \rho_{j}, \quad D_{3}^{2} g_{j}=g_{j} \gamma_{j-1}, \\
D_{4}^{2} g_{j}=(-1)^{j} g_{j} \gamma_{j-1}, \quad \partial_{2} g_{j}=g_{j}\left(c_{j}-c_{j-1}\right) .
\end{gathered}
$$

Now, using (2.3), (2.5), and (2.6), one can easily check that the bosonic and fermionic evolution derivatives satisfy the algebra of the $N=(2 \mid 2)$ supersymmetry

$$
\begin{gathered}
{\left[\partial_{a}, \partial_{b}\right]=\left[\partial_{a}, D_{s}^{b}\right]=0,} \\
\left\{D_{s}^{1}, D_{p}^{1}\right\}=(-1)^{s} 2 \delta_{s, p} \partial_{1}, \quad\left\{D_{s}^{2}, D_{p}^{2}\right\}=-(-1)^{s} 2 \delta_{s, p} \partial_{2},
\end{gathered}
$$

which can be realized via

$$
\partial_{a}=\frac{\partial}{\partial z_{a}}, \quad D_{s}^{1}=\frac{\partial}{\partial_{\theta_{s}}}+(-1)^{s} \theta_{s} \frac{\partial}{\partial z_{1}}, \quad D_{p}^{2}=\frac{\partial}{\partial_{\theta_{p}}}-(-1)^{p} \theta_{p} \frac{\partial}{\partial z_{2}},
$$

where $z_{a}(a=1,2)$ and $\theta_{s}, \theta_{p}(s=1,2 ; p=3,4)$ are the bosonic and fermionic evolution times of the $N=(2 \mid 2)$ superspace, respectively.

Looking at (2.5) and (2.6), one can see that they are not consistent with the boundary conditions (2.4c). Thus, it is impossible to simultaneously satisfy the boundary conditions for the fields $g_{j}$ entering into (2.5)

$$
\lim _{j \rightarrow \pm \infty} d_{2 j}=\lim _{j \rightarrow \pm \infty} g_{2 j} g_{2 j-1}=0, \quad \lim _{j \rightarrow \pm \infty} d_{2 j+1}=\lim _{j \rightarrow \pm \infty} g_{2 j+1} g_{2 j}=1,
$$

while (2.6) contain a contradiction at infinity in the equation for the field $\rho_{j}$. Thus, one can conclude that the boundary conditions strictly restrict the symmetries of (2.3). The periodic boundary conditions will be considered in Section 5.

Now we present other two related representations of the $2 \mathrm{D}$ generalized fermionic TL equations (2.3) which will be useful in what follows.

The first representation can be easily derived if one introduces a new basis $\left\{g_{j}, c_{j}\right.$, $\left.\gamma_{j}^{+}, \gamma_{j}^{-}\right\}$in the space of the fields $\left\{d_{j}, c_{j}, \gamma_{j}, \rho_{j}\right\}$,

$$
d_{j}=g_{j} g_{j-1}, \quad \rho_{j}=g_{j} \gamma_{j}^{-}, \quad \gamma_{j}=\gamma_{j+1}^{+},
$$

and eliminates the fields $c_{j}$ from (2.3) in order to get the conventional form of the $2 \mathrm{D}$ $N=(2 \mid 2)$ supersymmetric TL equations [5]

$$
\begin{gathered}
\partial_{1} \partial_{2} \ln g_{j}=g_{j+1} g_{j+2}-g_{j}\left(g_{j+1}+g_{j-1}\right)+g_{j-1} g_{j-2}+g_{j+1} \gamma_{j+1}^{+} \gamma_{j+1}^{-}-g_{j-1} \gamma_{j-1}^{+} \gamma_{j-1}^{-}, \\
\partial_{1} \gamma_{j}^{+}=g_{j+1} \gamma_{j+1}^{-}-g_{j-1} \gamma_{j-1}^{-}, \quad \partial_{2} \gamma_{j}^{-}=g_{j+1} \gamma_{j+1}^{+}-g_{j-1} \gamma_{j-1}^{+},
\end{gathered}
$$


together with their fermionic $N=(2 \mid 2)$ symmetries

$$
\begin{aligned}
D_{1}^{1} g_{j} & =g_{j} \gamma_{j}^{-}, & D_{2}^{1} g_{j} & =(-1)^{j} g_{j} \gamma_{j}^{-}, \\
D_{1}^{1} \gamma_{j}^{-} & =-\partial_{1} \ln g_{j}, & D_{2}^{1} \gamma_{j}^{-} & =(-1)^{j} \partial_{1} \ln g_{j}, \\
D_{1}^{1} \gamma_{j}^{+} & =g_{j-1}-g_{j+1}, & D_{2}^{1} \gamma_{j}^{+} & =(-1)^{j}\left(g_{j-1}-g_{j+1}\right), \\
D_{3}^{2} g_{j} & =g_{j} \gamma_{j}^{+}, & D_{4}^{2} g_{j} & =(-1)^{j} g_{j} \gamma_{j}^{+}, \\
D_{3}^{2} \gamma_{j}^{-} & =g_{j+1}-g_{j-1}, & D_{4}^{2} \gamma_{j}^{-} & =(-1)^{j}\left(g_{j+1}-g_{j-1}\right), \\
D_{3}^{2} \gamma_{j}^{+} & =\partial_{2} \ln g_{j}, & D_{4}^{2} \gamma_{j}^{+} & =-(-1)^{j} \partial_{2} \ln g_{j} .
\end{aligned}
$$

In order to derive the second representation, we introduce a new notation for the fields at odd and even values of the lattice coordinate $j$ :

$$
\begin{aligned}
& a_{j} \equiv c_{2 j+1}, \quad b_{j} \equiv d_{2 j+1}, \quad \alpha_{j} \equiv \gamma_{2 j-1}, \quad \beta_{j} \equiv \rho_{2 j+1}, \\
& \bar{a}_{j} \equiv c_{2 j}, \quad \bar{b}_{j} \equiv d_{2 j}, \quad \bar{\alpha}_{j} \equiv-\gamma_{2 j}, \quad \bar{\beta}_{j} \equiv \rho_{2 j},
\end{aligned}
$$

and rewrite (2.3), (2.5), and (2.6) in the following form:

$$
\begin{array}{lll}
\partial_{2} b_{j}=b_{j}\left(a_{j}-a_{j-1}\right), & \partial_{1} a_{j}=b_{j+1}-b_{j}+\beta_{j} \bar{\alpha}_{j}+\alpha_{j+1} \bar{\beta}_{j+1}, \\
\partial_{2} \bar{b}_{j}=\bar{b}_{j}\left(\bar{a}_{j}-\bar{a}_{j-1}\right), & \partial_{1} \bar{a}_{j}=\bar{b}_{j+1}-\bar{b}_{j}+\beta_{j} \bar{\alpha}_{j}+\alpha_{j} \bar{\beta}_{j}, \\
\partial_{1} \alpha_{j}=\beta_{j}-\beta_{j-1}, & \partial_{2} \beta_{j}=\left(a_{j}-\bar{a}_{j}\right) \beta_{j}-b_{j} \alpha_{j}+\bar{b}_{j+1} \alpha_{j+1}, \\
\partial_{1} \bar{\alpha}_{j}=\bar{\beta}_{j}-\bar{\beta}_{j+1}, & \partial_{2} \bar{\beta}_{j}=\left(\bar{a}_{j}-a_{j-1}\right) \bar{\beta}_{j}-b_{j} \bar{\alpha}_{j}+\bar{b}_{j} \alpha_{j-1}, \\
D_{1}^{1} b_{j}=e_{j} \bar{\beta}_{j}+\bar{e}_{j} \beta_{j}, & D_{2}^{1} b_{j}=-e_{j} \bar{\beta}_{j}-\bar{e}_{j} \beta_{j}, \\
D_{1}^{1} \bar{b}_{j}=e_{j-1} \bar{\beta}_{j}+\bar{e}_{j} \beta_{j-1}, & D_{2}^{1} \bar{b}_{j}=e_{j-1} \bar{\beta}_{j}-\bar{e}_{j} \beta_{j-1}, \\
D_{1}^{1} a_{j}=\bar{e}_{j+1} \alpha_{j+1}-e_{j} \bar{\alpha}_{j}, & D_{2}^{1} a_{j}=-\bar{e}_{j+1} \alpha_{j+1}-e_{j} \bar{\alpha}_{j}, \\
D_{1}^{1} \bar{a}_{j}=\bar{e}_{j} \alpha_{j}-e_{j} \bar{\alpha}_{j}, & D_{2}^{1} \bar{a}_{j}=-\bar{e}_{j} \alpha_{j}-e_{j} \bar{\alpha}_{j}, \\
D_{1}^{1} \beta_{j}=-\partial_{1} \bar{e}_{j}, & D_{2}^{1} \beta_{j}=-\partial_{1} \bar{e}_{j}, \\
D_{1}^{1} \bar{\beta}_{j}=-\partial_{1} \bar{e}_{j}, & D_{2}^{1} \bar{\beta}_{j}=\partial_{1} \bar{e}_{j}, \\
D_{1}^{1} \alpha_{j}=e_{j-1}-e_{j}, & D_{2}^{1} \alpha_{j}=e_{j-1}-e_{j}, \\
D_{1}^{1} \bar{\alpha}_{j}=\bar{e}_{j+1}-\bar{e}_{j}, & D_{2}^{1} \bar{\alpha}_{j}=\bar{e}_{j}-\bar{e}_{j+1}, \\
D_{3}^{2} b_{j}=b_{j}\left(\alpha_{j}-\bar{\alpha}_{j}\right), & D_{4}^{2} b_{j}=b_{j}\left(\alpha_{j}+\bar{\alpha}_{j}\right), \\
D_{3}^{2} \bar{b}_{j}=\bar{b}_{j}\left(\alpha_{j}-\bar{\alpha}_{j-1}\right), & D_{4}^{2} \bar{b}_{j}=\bar{b}_{j}\left(\alpha_{j}+\bar{\alpha}_{j-1}\right), \\
D_{3}^{2} a_{j}=\partial_{2} \sum_{k=-\infty}\left(\alpha_{k}-\bar{\alpha}_{k}\right), & D_{4}^{2} a_{j}=\partial_{2} \sum_{k=-\infty}\left(\alpha_{k}+\bar{\alpha}_{k}\right), \\
& &
\end{array}
$$


118 Generalized fermionic discrete Toda hierarchy

$$
\begin{array}{ll}
D_{3}^{2} \bar{a}_{j}=\partial_{2} \sum_{k=-\infty}^{j}\left(\alpha_{k}-\bar{\alpha}_{k-1}\right), & D_{4}^{2} \bar{a}_{j}=\partial_{2} \sum_{k=-\infty}^{j}\left(\alpha_{k}+\bar{\alpha}_{k-1}\right), \\
D_{3}^{2} \beta_{j}=\bar{b}_{j}-b_{j}-\bar{b}_{i}+\beta_{j} \bar{\alpha}_{j}, & D_{4}^{2} 1 \beta_{j}=b_{j}-\bar{b}_{j+1}-\beta_{j} \bar{\alpha}_{j}, \\
D_{3}^{2} \bar{\beta}_{j}=b_{j}-\bar{b}_{j}-\bar{\beta}_{j} \alpha_{j}, & D_{4}^{2} \bar{\beta}_{j}=b_{j}-\bar{b}_{j}-\bar{\beta}_{j} \alpha_{j}, \\
D_{3}^{2} \alpha_{j}=\bar{a}_{j}-a_{j-1}, & D_{4}^{2} \alpha_{j}=a_{j-1}-\bar{a}_{j}, \\
D_{3}^{2} \bar{\alpha}_{j}=\bar{a}_{j}-a_{j}, & D_{4}^{2} \bar{\alpha}_{j}=\bar{a}_{j}-a_{j},
\end{array}
$$

where $e_{j}$ and $\bar{e}_{j}$ are the composite fields

$$
e_{j} \equiv g_{2 j+1} \equiv \prod_{k=0}^{\infty} \frac{b_{j-k}}{\bar{b}_{j-k}}, \quad \bar{e}_{j} \equiv g_{2 j} \equiv \prod_{k=0}^{\infty} \frac{\bar{b}_{j-k}}{b_{j-k-1}},
$$

which obey the equations

$$
\begin{gathered}
\partial_{2} e_{j}=e_{j}\left(a_{j}-\bar{a}_{j}\right), \quad \partial_{2} \bar{e}_{j}=\bar{e}_{j}\left(\bar{a}_{j}-a_{j-1}\right), \\
D_{1}^{1} e_{j}=\beta_{j}, \quad D_{2}^{1} e_{j}=-\beta_{j}, \quad D_{3}^{2} e_{j}=e_{j} \alpha_{j},
\end{gathered}
$$

The reduction

$$
\bar{b}_{j}=0
$$

of (2.16) leads to the $2 \mathrm{D} N=(0 \mid 2)$ supersymmetric TL equations $[5,11]$. One can easily see that fermionic symmetries (2.17) are not consistent with this reduction, while fermionic symmetries (2.18) are consistent and form the algebra of the $N=(0 \mid 2)$ supersymmetry.

2.2. Bi-Hamiltonian structure of the $1 \mathrm{D}$ generalized fermionic TL hierarchy. Our further purpose is to construct a bi-Hamiltonian structure of the generalized fermionic TL equations (2.3) (consequently, originating from them (2.13) and (2.16)) in 1D space when all the fields depend on only one bosonic coordinate $z=z_{1}+z_{2}$. This task was solved in [3] for the 1D $N=2$ TL hierarchy obtained by reduction (2.21) of the $1 \mathrm{D}$ generalized fermionic TL hierarchy. Here we solve this task for the original 1D generalized fermionic TL hierarchy.

At the reduction to $1 \mathrm{D}$ space,

$$
\partial_{1}=\partial_{2} \equiv \partial
$$


the zero-curvature representation (2.1) can be identically rewritten in the form of the Lax-pair representation

$$
L=\left(\begin{array}{cccccccc}
\cdots & \partial=\left[L, L^{-}\right], & L \equiv L^{+}+L^{-}, & \\
\cdots & c_{j} & \gamma_{j} & 1 & 0 & 0 & 0 & \cdots \\
& \rho_{j+1} & c_{j+1} & \gamma_{j+1} & 1 & 0 & 0 & \\
& d_{j+2} & \rho_{j+2} & c_{j+2} & \gamma_{j+2} & 1 & 0 & \\
& 0 & d_{j+3} & \rho_{j+3} & c_{j+3} & \gamma_{j+3} & 1 & \\
& 0 & 0 & d_{j+4} & \rho_{j+4} & c_{j+4} & \gamma_{j+4} & \\
\cdots & 0 & 0 & 0 & d_{j+5} & \rho_{j+5} & c_{j+5} & \cdots \\
\cdots & \cdots & & & & & \cdots & \cdots
\end{array}\right) .
$$

Using the Lax-pair representation (2.23), it is easy to derive the general expression for bosonic Hamiltonians which are in involution via the standard formula

$$
H_{k}=\frac{1}{k} \operatorname{str} L^{k} \equiv \frac{1}{k} \sum_{p=1}^{\infty}(-1)^{p}\left(L^{k}\right)_{p p} .
$$

The first two of them have the following explicit form:

$$
H_{1}=\sum_{i=-\infty}^{\infty}(-1)^{i} c_{i}, \quad H_{2}=\sum_{i=-\infty}^{\infty}(-1)^{i}\left(\frac{1}{2} c_{i}^{2}+d_{i}+\rho_{i} \gamma_{i-1}\right) .
$$

A bi-Hamiltonian system of evolution equations can be represented in the following general form:

$$
\frac{\partial}{\partial t_{H_{k}}} q_{i}=\left\{H_{k+1}, q_{i}\right\}_{1}=\left\{H_{k}, q_{i}\right\}_{2}
$$

where $t_{H_{k}}$ are the evolution times, $q_{j}$ denotes any field from the set $q_{i}=\left\{d_{i}, c_{i}, \rho_{i}, \gamma_{i}\right\}$, and the brackets $\{\cdot, \cdot\}_{1(2)}$ are appropriate Poisson brackets corresponding to the first (second) Hamiltonian structure. Using (2.26) and the 2D generalized fermionic TL equations (2.3) at the reduction to $1 \mathrm{D}$ space (2.22) — the $1 \mathrm{D}$ generalized fermionic TL equations

$$
\begin{array}{ll}
\partial d_{i}=d_{i}\left(c_{i}-c_{i-2}\right), & \partial c_{i}=d_{i+2}-d_{i}+\gamma_{i} \rho_{i+1}+\gamma_{i-1} \rho_{i}, \\
\partial \gamma_{i}=\rho_{i+2}-\rho_{i}, & \partial \rho_{i}=\rho_{i}\left(c_{i}-c_{i-1}\right)+d_{i+1} \gamma_{i}-d_{i} \gamma_{i-2}
\end{array}
$$

as well as Hamiltonians (2.25), we have found the first two Hamiltonian structures of the hierarchy. As a result, we have the following explicit expressions:

$$
\begin{aligned}
& \left\{d_{i}, c_{j}\right\}_{1}=(-1)^{j} d_{i}\left(\delta_{i, j+2}-\delta_{i, j}\right), \\
& \left\{c_{i}, \rho_{j}\right\}_{1}=(-1)^{j} \rho_{j}\left(\delta_{i, j-1}+\delta_{i, j}\right), \\
& \left\{\rho_{i}, \rho_{j}\right\}_{1}=(-1)^{j}\left(d_{i} \delta_{i, j+1}-d_{j} \delta_{i, j-1}\right), \\
& \left\{\gamma_{i}, \gamma_{j}\right\}_{1}=(-1)^{j}\left(\delta_{i, j+1}-\delta_{i, j-1}\right)
\end{aligned}
$$


for the first and

$$
\begin{aligned}
& \left\{d_{i}, d_{j}\right\}_{2}=(-1)^{j} d_{i} d_{j}\left(\delta_{i, j+2}-\delta_{i, j-2}\right), \\
& \left\{d_{i}, c_{j}\right\}_{2}=(-1)^{j} d_{i} c_{j}\left(\delta_{i, j+2}-\delta_{i, j}\right), \\
& \left\{c_{i}, c_{j}\right\}_{2}=(-1)^{j}\left(d_{i} \delta_{i, j+2}-d_{j} \delta_{i, j-2}-\gamma_{j} \rho_{i} \delta_{i, j+1}-\gamma_{i} \rho_{j} \delta_{i, j-1}\right), \\
& \left\{d_{i}, \rho_{j}\right\}_{2}=(-1)^{j} d_{i} \rho_{j}\left(\delta_{i, j+2}+\delta_{i, j-1}\right), \\
& \left\{d_{i}, \gamma_{j}\right\}_{2}=(-1)^{j} d_{i} \gamma_{j}\left(\delta_{i, j+2}+\delta_{i, j+1}\right), \\
& \left\{c_{i}, \rho_{j}\right\}_{2}=(-1)^{j}\left(c_{i} \rho_{j}\left(\delta_{i, j}+\delta_{i, j-1}\right)-d_{j} \gamma_{i} \delta_{i, j-2}-d_{i} \gamma_{j} \delta_{i, j+1}\right), \\
& \left\{c_{i}, \gamma_{j}\right\}_{2}=(-1)^{j}\left(\rho_{i} \delta_{i, j+2}+\rho_{j} \delta_{i, j-1}\right), \\
& \left\{\rho_{i}, \gamma_{j}\right\}_{2}=(-1)^{j}\left(\rho_{i} \gamma_{j} \delta_{i, j+1}+d_{i} \delta_{i, j+3}-d_{j} \delta_{i, j-1}\right), \\
& \left\{\rho_{i}, \rho_{j}\right\}_{2}=(-1)^{j}\left(\left(\rho_{i} \rho_{j}-d_{j} c_{i}\right) \delta_{i, j-1}+\left(\rho_{i} \rho_{j}+d_{i} c_{j}\right) \delta_{i, j+1}\right), \\
& \left\{\gamma_{i}, \gamma_{j}\right\}_{2}=(-1)^{j}\left(c_{i} \delta_{i, j+1}-c_{j} \delta_{i, j-1}\right)
\end{aligned}
$$

for the second Hamiltonian structures, where only nonzero brackets are written down.

Note that the first $\{\cdot, \cdot\}_{1}$ in (2.28) and the second $\{\cdot, \cdot\}_{2}$ in (2.29) Hamiltonian structures are obviously compatible: the deformation of the fields $c_{j} \rightarrow c_{j}+v$, where $v$ is an arbitrary constant, transforms $\{\cdot, \cdot\}_{2}$ into the Hamiltonian structure which is their sum

$$
\{\cdot, \cdot\}_{2} \longrightarrow\{\cdot, \cdot\}_{2}+\nu\{\cdot, \cdot\}_{1}
$$

Thus, one concludes that the corresponding recursion operator

$$
R=\{\cdot, \cdot\}_{2}\{\cdot, \cdot\}_{1}^{-1}
$$

is hereditary like the operator obtained from the compatible pair of Hamiltonian structures.

We have checked that the $1 \mathrm{D}$ reduction (2.22) of the fermionic symmetries (2.5) and (2.6),

$$
\begin{array}{lll}
D_{1} d_{i}=g_{i-1} \rho_{i}+g_{i} \rho_{i-1}, & D_{2} d_{i}=(-1)^{i}\left(g_{i-1} \rho_{i}-g_{i} \rho_{i-1}\right), \\
D_{1} c_{i}=g_{i} \gamma_{i-1}+g_{i+1} \gamma_{i}, & D_{2} c_{i}=(-1)^{i}\left(g_{i+1} \gamma_{i}-g_{i} \gamma_{i-1}\right), \\
D_{1} \rho_{i}=g_{i}\left(c_{i-1}-c_{i}\right), & D_{2} \rho_{i}=(-1)^{i} g_{i}\left(c_{i}-c_{i-1}\right), \\
D_{1} \gamma_{i}=g_{i}-g_{i+2}, & D_{2} \gamma_{i}=(-1)^{i}\left(g_{i+2}-g_{i}\right), \\
D_{3} d_{i}=d_{i}\left(\gamma_{i-1}+\gamma_{i-2}\right), & D_{4} d_{i}=(-1)^{i} d_{i}\left(\gamma_{i-1}-\gamma_{i-2}\right), \\
D_{3} c_{i}=\rho_{i+1}+\rho_{i}, & D_{4} c_{i}=(-1)^{i}\left(\rho_{i+1}-\rho_{i}\right), \\
D_{3} \rho_{i}=d_{i+1}-d_{i}-\rho_{i} \gamma_{i-1}, & D_{4} \rho_{i}=(-1)^{i}\left(d_{i+1}-d_{i}-\rho_{i} \gamma_{i-1}\right), \\
D_{3} \gamma_{i}=c_{i+1}-c_{i}, & D_{4} \gamma_{i}=(-1)^{i}\left(c_{i+1}-c_{i}\right),
\end{array}
$$

and the equations for the composite fields $g_{j}(2.7)$,

$$
\begin{gathered}
\partial g_{j}=g_{j}\left(c_{j}-c_{j-1}\right), \quad D_{1} g_{j}=\rho_{j}, \quad D_{2} g_{j}=(-1)^{j} \rho_{j}, \\
D_{3} g_{j}=g_{j} \gamma_{j-1}, \quad D_{4} g_{j}=(-1)^{j} g_{j} \gamma_{j-1},
\end{gathered}
$$


can also be represented in a bi-Hamiltonian form with fermionic Hamiltonians $S_{s, k}$ and Hamiltonian structures (2.28) and (2.29),

$$
D_{t_{s, k}} q_{i}=\left\{S_{s, k+1}, q_{i}\right\}_{1}=\left\{S_{s, k}, q_{i}\right\}_{2},
$$

where $D_{t_{s, k}}$ are the fermionic evolution derivatives. In Section 3.2, we show how fermionic Hamiltonians can be derived in an algorithmic way, but now we only mention that there are four infinite towers of fermionic Hamiltonians $S_{s, k}(s=1,2,3,4 ; k \in \mathbb{N})$ and present without any comments only explicit expressions for the first few of them:

$$
\begin{array}{ll}
S_{1,1}=\sum_{i=-\infty}^{\infty}(-1)^{i} \rho_{i} g_{i}^{-1}, & S_{1,2}=-\sum_{i=-\infty}^{\infty}\left((-1)^{i} g_{i} \gamma_{i-1}+\rho_{i} g_{i}^{-1} \sum_{j=-\infty}^{i-1}(-1)^{j} c_{j}\right), \\
S_{2,1}=\sum_{i=-\infty}^{\infty} \rho_{i} g_{i}^{-1}, & S_{2,2}=\sum_{i=-\infty}^{\infty}\left(g_{i} \gamma_{i-1}-(-1)^{i} \rho_{i} g_{i}^{-1} \sum_{j=-\infty}^{i-1}(-1)^{j} c_{j}\right), \\
S_{3,1}=-\sum_{i=-\infty}^{\infty}(-1)^{i} \gamma_{i}, & S_{3,2}=-\sum_{i=-\infty}^{\infty}\left((-1)^{i} \rho_{i}+\gamma_{i-1} \sum_{j=-\infty}^{i-1}(-1)^{j} c_{j}\right), \\
S_{4,1}=\sum_{i=-\infty}^{\infty} \gamma_{i}, & S_{4,2}=\sum_{i=-\infty}^{\infty}\left(\rho_{i}-(-1)^{i} \gamma_{i-1} \sum_{j=-\infty}^{i-1}(-1)^{j} c_{j}\right) .
\end{array}
$$

For completeness, we also present the nonzero Poisson brackets of the composite field $g_{j}$ (2.7) with other fields of the hierarchy which are useful when producing fermionic Hamiltonian flows

$$
\begin{aligned}
& \left\{g_{i}, c_{j}\right\}_{1}=(-1)^{j} g_{i}\left(\delta_{i, j+1}-\delta_{i, j}\right), \\
& \left\{g_{i}, \gamma_{j}\right\}_{2}=(-1)^{j} g_{i} \gamma_{j} \delta_{i, j+1}, \\
& \left\{g_{i}, c_{j}\right\}_{2}=(-1)^{j} g_{i} c_{j}\left(\delta_{i, j+1}-\delta_{i, j}\right), \\
& \left\{g_{i}, \rho_{j}\right\}_{2}=(-1)^{j} g_{i} \rho_{j}\left(\delta_{i, j+1}-\delta_{i, j}+\delta_{i, j-1}\right), \\
& \left\{g_{i}, d_{j}\right\}_{2}=(-1)^{j} g_{i} d_{j}\left(\delta_{i, j+1}-\delta_{i, j}+\delta_{i, j-1}-\delta_{i, j-2}\right), \\
& \left\{g_{i}, g_{j}\right\}_{2}=(-1)^{j} g_{i} g_{j}\left(\delta_{i, j+1}+\delta_{i, j-1}\right) .
\end{aligned}
$$

Now we have all the necessary ingredients to derive Hamiltonian flows of the 1D generalized TL hierarchy. We end this section with a few remarks.

First, the Hamiltonians $H_{1}$ in (2.25) and $S_{s, 1}$ in (2.35) give trivial flows via the first Hamiltonian structure (2.28) because they belong to the center of the algebra (2.28),

$$
\left\{H_{1}, q_{j}\right\}_{1}=\left\{S_{s, 1}, q_{j}\right\}_{1}=0 \text {. }
$$

Second, while the densities corresponding to the fermionic Hamiltonians $S_{p, k}$ in (2.35) have a nonlocal character with respect to the lattice indices, the fermionic flows (2.32) have no nonlocal terms. 
Finally, the algebras of the first and second Hamiltonian structures (2.28) and (2.29) together with (2.36) possess a discrete inner automorphism $f$ which transforms nontrivially only fermionic fields,

$$
\gamma_{j} \stackrel{f}{\longmapsto}(-1)^{j} \rho_{j+1} g_{j+1}^{-1}, \quad \rho_{j} \stackrel{f}{\longmapsto}(-1)^{j} \gamma_{j-1} g_{j} .
$$

Using (2.33), one can easily check that the automorphism $f$ transforms (2.27), (2.32), and Hamiltonians (2.25) and (2.35) according to the following rule:

$$
\begin{gathered}
\left\{\partial, D_{1}, D_{2}, D_{3}, D_{4}\right\} \stackrel{f}{\longmapsto}\left\{\partial, D_{4}, D_{3},-D_{2},-D_{1}\right\}, \\
\left\{H_{k}, S_{1, k}, S_{2, k}, S_{3, k}, S_{4, k}\right\} \stackrel{f}{\longmapsto}\left\{H_{k},-S_{4, k},-S_{3, k},-S_{2, k},-S_{1, k}\right\} .
\end{gathered}
$$

\section{Reduction: $1 \mathrm{D} N=4$ supersymmetric TL hierarchy}

3.1. Bi-Hamiltonian structure of the $1 \mathrm{D} N=4 \mathrm{TL}$ hierarchy. In this section, we consider the bi-Hamiltonian formulation of the $1 \mathrm{D}$ reduction $(2.22)$ of the $2 \mathrm{D} \mathrm{N}=(2 \mid 2)$ supersymmetric TL equations (2.13) and their fermionic symmetries (2.14). Starting with Hamiltonians (2.25) and (2.35) and Hamiltonian structures (2.28) and (2.29) as well as using relations (2.12), it is easy to represent (2.13) in 1D space as a bi-Hamiltonian system of first-order evolution equations.

Thus, we obtain the following bosonic and fermionic Hamiltonians:

$$
\begin{array}{rlrl}
H_{1}^{N=4} & =\sum_{i=-\infty}^{\infty}(-1)^{i} c_{i}, & H_{2}^{N=4} & =\sum_{i=-\infty}^{\infty}(-1)^{i}\left(\frac{1}{2} c_{i}^{2}+g_{i} g_{i-1}+g_{i} \gamma_{i}^{-} \gamma_{i}^{+}\right), \\
S_{1,1}^{N=4} & =\sum_{i=-\infty}^{\infty}(-1)^{i} \gamma_{i}^{-}, & S_{1,2}^{N=4}=-\sum_{i=-\infty}^{\infty}\left((-1)^{i} g_{i} \gamma_{i}^{+}+\gamma_{i}^{-} \sum_{k=-\infty}^{i-1}(-1)^{k} c_{k}\right), \\
S_{2,1}^{N=4}=\sum_{i=-\infty}^{\infty} \gamma_{i}^{-}, & S_{2,2}^{N=4}=\sum_{i=-\infty}^{\infty}\left(g_{i} \gamma_{i}^{+}-(-1)^{i} \gamma_{i}^{-} \sum_{k=-\infty}^{i-1}(-1)^{k} c_{k}\right), \\
S_{3,1}^{N=4}=\sum_{i=-\infty}^{\infty}(-1)^{i} \gamma_{i}^{+}, & S_{3,2}^{N=4}=-\sum_{i=-\infty}^{\infty}\left((-1)^{i} g_{i} \gamma_{i}^{-}+\gamma_{i}^{+} \sum_{k=-\infty}^{i-1}(-1)^{k} c_{k}\right), \\
S_{4,1}^{N=4}=\sum_{i=-\infty}^{\infty} \gamma_{i}^{+}, & S_{4,2}^{N=4}=\sum_{i=-\infty}^{\infty}\left(g_{i} \gamma_{i}^{-}-(-1)^{i} \gamma_{i}^{+} \sum_{k=-\infty}^{i-1}(-1)^{k} c_{k}\right),
\end{array}
$$

the first Hamiltonian structure

$$
\begin{gathered}
\left\{\gamma_{i}^{ \pm}, \gamma_{j}^{ \pm}\right\}_{1}= \pm(-1)^{j}\left(\delta_{i, j-1}-\delta_{i, j+1}\right), \\
\left\{g_{i}, c_{j}\right\}_{1}=(-1)^{j} g_{i}\left(\delta_{i, j+1}-\delta_{i, j}\right),
\end{gathered}
$$


and the second Hamiltonian structure

$$
\begin{aligned}
\left\{g_{i}, g_{j}\right\}_{2} & =(-1)^{j} g_{i} g_{j}\left(\delta_{i, j+1}+\delta_{i, j-1}\right), \\
\left\{g_{i}, \gamma_{j}^{ \pm}\right\}_{2} & =-(-1)^{j} g_{i} \gamma_{j}^{ \pm} \delta_{i, j}, \\
\left\{\gamma_{i}^{ \pm}, \gamma_{j}^{ \pm}\right\}_{2} & = \pm(-1)^{j}\left(c_{i} \delta_{i, j-1}-c_{j} \delta_{i, j+1}\right), \\
\left\{\gamma_{i}^{-}, \gamma_{j}^{+}\right\}_{2} & =(-1)^{j}\left(g_{i+1} \delta_{i, j-2}-g_{i-1} \delta_{i, j+2}\right), \\
\left\{c_{i}, c_{j}\right\}_{2} & =(-1)^{j}\left(g_{i} g_{i-1} \delta_{i, j+2}-g_{j} g_{j-1} \delta_{i, j-2}-g_{i} \gamma_{i}^{+} \gamma_{i}^{-} \delta_{i, j+1}-g_{j} \gamma_{j}^{+} \gamma_{j}^{-} \delta_{i, j-1}\right), \\
\left\{g_{i}, c_{j}\right\}_{2} & =(-1)^{j} g_{i} c_{j}\left(\delta_{i, j+1}-\delta_{i, j}\right), \\
\left\{c_{i}, \gamma_{j}^{ \pm}\right\}_{2} & =-(-1)^{j}\left(g_{i} \gamma_{i}^{\mp} \delta_{i, j+1}+g_{j-1} \gamma_{j-1}^{\mp} \delta_{i, j-2}\right),
\end{aligned}
$$

where only nonzero brackets are presented. For the first nontrivial bosonic and fermionic flows, one obtains in a standard way, using (2.26), (2.34), (3.1), (3.2), and (3.3),

$$
\begin{aligned}
\partial g_{i} & =g_{i}\left(c_{i}-c_{i-1}\right), & \partial c_{i} & =-g_{i-1} g_{i}+g_{i+1} g_{i+2}+g_{i+1} \gamma_{i+1}^{+} \gamma_{i+1}^{-}+g_{i} \gamma_{i}^{+} \gamma_{i}^{-}, \\
\partial \gamma_{i}^{+} & =g_{i+1} \gamma_{i+1}^{-}-g_{i-1} \gamma_{i-1}^{-}, & \partial \gamma_{i}^{-} & =g_{i+1} \gamma_{i+1}^{+}-g_{i-1} \gamma_{i-1}^{+}, \\
D_{1} g_{i} & =g_{i} \gamma_{i}^{-}, & D_{2} g_{i} & =(-1)^{i} g_{i} \gamma_{i}^{-}, \\
D_{1} \gamma_{i}^{-} & =c_{i-1}-c_{i}, & D_{2} \gamma_{i}^{-} & =(-1)^{i}\left(c_{i}-c_{i-1}\right), \\
D_{1} \gamma_{i}^{+} & =g_{i-1}-g_{i+1}, & D_{2} \gamma_{i}^{+} & =(-1)^{i}\left(g_{i-1}-g_{i+1}\right), \\
D_{1} c_{i} & =g_{i+1} \gamma_{i+1}^{+}+g_{i} \gamma_{i}^{+}, & D_{2} c_{i} & =(-1)^{i}\left(g_{i+1} \gamma_{i+1}^{+}-g_{i} \gamma_{i}^{+}\right), \\
D_{3} g_{i} & =g_{i} \gamma_{i}^{+}, & D_{4} g_{i} & =(-1)^{i} g_{i} \gamma_{i}^{+}, \\
D_{3} \gamma_{i}^{-} & =g_{i+1}-g_{i-1}, & D_{4} \gamma_{i}^{-} & =(-1)^{i}\left(g_{i+1}-g_{i-1}\right), \\
D_{3} \gamma_{i}^{+} & =c_{i}-c_{i-1}, & D_{4} \gamma_{i}^{+} & =(-1)^{i}\left(c_{i-1}-c_{i}\right), \\
D_{3} c_{i} & =g_{i+1} \gamma_{i+1}^{-}+g_{i} \gamma_{i}^{-}, & D_{4} c_{i} & =(-1)^{i}\left(g_{i+1} \gamma_{i+1}^{-}-g_{i} \gamma_{i}^{-}\right) .
\end{aligned}
$$

For the system (3.4), we consider the boundary conditions at infinity of the following two types:

$$
\begin{array}{lll}
\lim _{j \rightarrow \pm \infty} g_{j}=0, & \lim _{j \rightarrow \pm \infty} c_{j}=0, & \lim _{j \rightarrow \pm \infty} \gamma_{j}^{ \pm}=0 ; \\
\lim _{j \rightarrow \pm \infty} g_{j}=1, & \lim _{j \rightarrow \pm \infty} c_{j}=0, & \lim _{j \rightarrow \pm \infty} \gamma_{j}^{ \pm}=0,
\end{array}
$$

which are the consequences of the boundary conditions (2.4a) and (2.4b). Flows (3.4), (3.5), and (3.6) are compatible with these boundary conditions and form the $N=4$ supersymmetry algebra. 
The fields $c_{i}$ can be dropped out of the system (3.4) and finally (3.4) takes the form of (2.13) in $1 \mathrm{D}$ space $(2.22)$,

$$
\begin{gathered}
\partial^{2} \ln g_{i}=g_{i+1} g_{i+2}-g_{i}\left(g_{i+1}+g_{i-1}\right)+g_{i-1} g_{i-2}+g_{i+1} \gamma_{i+1}^{+} \gamma_{i+1}^{-}-g_{i-1} \gamma_{i-1}^{+} \gamma_{i-1}^{-}, \\
\partial \gamma_{i}^{+}=g_{i+1} \gamma_{i+1}^{-}-g_{i-1} \gamma_{i-1}^{-}, \quad \partial \gamma_{i}^{-}=g_{i+1} \gamma_{i+1}^{+}-g_{i-1} \gamma_{i-1}^{+},
\end{gathered}
$$

with the $N=4$ supersymmetry transformations

$$
\begin{array}{rlrl}
D_{1} g_{i} & =g_{i} \gamma_{i}^{-}, & D_{2} g_{i} & =(-1)^{i} g_{i} \gamma_{i}^{-}, \\
D_{1} \gamma_{i}^{-} & =-\partial \ln g_{i}, & D_{2} \gamma_{i}^{-}=(-1)^{i} \partial \ln g_{i}, \\
D_{1} \gamma_{i}^{+}=g_{i-1}-g_{i+1}, & D_{2} \gamma_{i}^{+}=(-1)^{i}\left(g_{i-1}-g_{i+1}\right), \\
D_{3} g_{i}=g_{i} \gamma_{i}^{+}, & D_{4} g_{i}=(-1)^{i} g_{i} \gamma_{i}^{+}, \\
D_{3} \gamma_{i}^{-}=g_{i+1}-g_{i-1}, & D_{4} \gamma_{i}^{-}=(-1)^{i}\left(g_{i+1}-g_{i-1}\right), \\
D_{3} \gamma_{i}^{+}=\partial \ln g_{i}, & D_{4} \gamma_{i}^{+}=-(-1)^{i} \partial \ln g_{i} .
\end{array}
$$

Thus, (3.8) reproduce the $1 \mathrm{D} N=4$ supersymmetric TL equations.

In terms of the new variables (2.12), the automorphism $f$ in (2.38) becomes

$$
\gamma_{j}^{-} \stackrel{f}{\longmapsto}(-1)^{j} \gamma_{j}^{+}, \quad \gamma_{j}^{+} \stackrel{f}{\longmapsto}-(-1)^{j} \gamma_{j}^{-},
$$

and it transforms the flows (3.4), (3.5), and (3.6) and Hamiltonians (3.1) as follows:

$$
\begin{gathered}
\left\{\partial, D_{1}, D_{2}, D_{3}, D_{4}\right\} \stackrel{f}{\longmapsto}\left\{\partial, D_{4}, D_{3},-D_{2},-D_{1}\right\}, \\
\left\{H_{k}^{N=4}, S_{1, k}^{N=4}, S_{2, k}^{N=4}, S_{3, k}^{N=4}, S_{4, k}^{N=4}\right\} \stackrel{f}{\longmapsto}\left\{H_{k}^{N=4}, S_{4, k}^{N=4}, S_{3, k}^{N=4},-S_{2, k}^{N=4},-S_{1, k}^{N=4}\right\} .
\end{gathered}
$$

3.2. Fermionic Hamiltonians. The above-described 1D $N=4$ supersymmetric TL hierarchy is a bi-Hamiltonian system, and it includes both bosonic and fermionic flows which are generated via bosonic and fermionic Hamiltonians. The bosonic Hamiltonians are produced by means of formula (2.24), while the origin of the fermionic Hamiltonians is rather mysterious so far. In this section, we deduce general expressions generating fermionic Hamiltonians.

The $N=(2 \mid 2)$ TL equations (2.13) can be derived as a subsystem of the more general $N=(1 \mid 1)$ 2D supersymmetric TL hierarchy defined via the following Lax-pair representation [12]: 


$$
\begin{gathered}
D_{n}^{ \pm}\left(L^{\alpha}\right)_{*}^{m}=\mp \alpha(-1)^{n m}\left[\left(\left(\left(L^{ \pm}\right)_{*}^{n}\right)_{-\alpha}\right)^{*(m)},\left(L^{\alpha}\right)_{*}^{m}\right\}, \quad \alpha=+,-, n, m \in \mathbb{N}, \\
\left(L^{\alpha}\right)_{*}^{2 m} \equiv\left(\frac{1}{2}\left[\left(L^{\alpha}\right)^{*},\left(L^{\alpha}\right)\right\}\right)^{m}, \quad\left(L^{\alpha}\right)_{*}^{2 m+1} \equiv L^{\alpha}\left(L^{\alpha}\right)_{*}^{2 m}, \\
L^{+}=\sum_{k=0}^{\infty} u_{k, j} e^{(1-k) \partial}, \quad u_{0, j}=1, \quad L^{-}=\sum_{k=0}^{\infty} v_{k, j} e^{(k-1) \partial}, \\
\left(L^{+}\right)^{*}=\sum_{k=0}^{\infty}(-1)^{k} u_{k, j} e^{(1-k) \partial}, \quad\left(L^{-}\right)^{*}=\sum_{k=0}^{\infty}(-1)^{k} v_{k, j} e^{(k-1) \partial}, \\
d_{\left(L^{ \pm}\right)_{*}^{2 m+1}}=1, \quad d_{\left(L^{ \pm}\right)_{*}^{2 m}}=0 .
\end{gathered}
$$

All details concerning the $N=(1 \mid 1)$ 2DTL hierarchy can be found in $[11,12]$, here we only explain the notation. The generalized graded bracket operation $[\cdot, \cdot\}$, entering into (3.12), on the space of operators $\mathbb{O}$ with the grading $d_{\mathbb{O}}$ and the involution $*$, is defined as

$$
\left[\mathbb{O}_{1}, \mathbb{O}_{2}\right\}:=\mathbb{O}_{1} \mathbb{O}_{2}-(-1)^{d_{\mathbb{Q}_{1}} d_{\mathbb{O}_{2}} \mathbb{O}_{2}} *\left(d_{\mathbb{O}_{1}}\right) \mathbb{O}_{1} *\left(d_{\mathbb{O}_{2}}\right)
$$

where $\mathbb{O}^{*(m)}$ denotes the $m$-fold action of the involution $*$ on the operator $\mathbb{O}$. Equations (3.12) are written for the composite Lax operators

$$
\left(L^{+}\right)_{*}^{m}=\sum_{k=0}^{\infty} u_{k, j}^{(m)} e^{(m-k) \partial}, \quad u_{0, j}^{(m)}=1, \quad\left(L^{-}\right)_{*}^{m}=\sum_{k=0}^{\infty} v_{k, j}^{(m)} e^{(k-m) \partial}
$$

where $u_{k, j}^{(m)}$ and $v_{k, j}^{(m)}$ (with $u_{k, j}^{(1)} \equiv u_{k, j}, v_{k, j}^{(1)} \equiv v_{k, j}$ ) are the functionals of the original bosonic $u_{2 k, j}, v_{2 k, j}$ and fermionic $u_{2 k+1, j}, v_{2 k+1, j}$ lattice fields which parameterize the Lax operators $L^{ \pm}$in (3.12). The operator $e^{l \partial}(l \in \mathbb{Z})$ acts on these fields as the discrete lattice shift

$$
e^{l \partial} u_{k, j}^{(m)} \equiv u_{k, j+l}^{(m)} e^{l \partial}, \quad e^{l \partial} v_{k, j}^{(m)} \equiv v_{k, j+l}^{(m)} e^{l \partial}
$$

and the subscript $+(-)$ in (3.12) means the part of the corresponding operators which includes the operators $e^{l \partial}$ at $l \geq 0(l<0)$. The explicit form for the functionals $u_{k, j}^{(m)}$ and $v_{k, j}^{(m)}$ can be obtained through the representation of the composite Lax operators $\left(L^{ \pm}\right)_{*}^{m}$ in (3.12) and (3.14) in terms of the Lax operators $L^{ \pm}$in (3.12). The fields $u_{k, j}$ and $v_{k, j}$ depend on the bosonic $t_{2 n}^{ \pm}$and fermionic $t_{2 n+1}^{ \pm}$times, and $D_{2 n}^{ \pm}\left(D_{2 n+1}^{ \pm}\right)$in (3.12) means bosonic (fermionic) evolution derivatives with the algebra,

$$
\left[D_{n}^{+}, D_{l}^{-}\right\}=\left[D_{n}^{ \pm}, D_{2 l}^{ \pm}\right]=0, \quad\left\{D_{2 n+1}^{ \pm}, D_{2 l+1}^{ \pm}\right\}=2 D_{2(n+l+1)}^{ \pm}
$$

which can be realized via

$$
D_{2 n}^{ \pm}=\partial_{2 n}^{ \pm}, \quad D_{2 n+1}^{ \pm}=\partial_{2 n+1}^{ \pm}+\sum_{l=1}^{\infty} t_{2 l-1}^{ \pm} \partial_{2(k+l)}^{ \pm}, \quad \partial_{n}^{ \pm}=\frac{\partial}{\partial t_{n}^{ \pm}}
$$


Now using the above-described definitions, one can derive flows for the functionals $u_{k, j}^{(m)}$ and $v_{k, j}^{(m)}$ corresponding to the Lax-pair representation (3.12). Thus, we obtain $[11,12]$

$$
\begin{aligned}
D_{n}^{+} u_{k, j}^{(2 m)}= & \sum_{p=0}^{n}\left(u_{p, j}^{(n)} u_{k-p+n, j-p+n}^{(2 m)}-(-1)^{(p+n)(k-p+n)} u_{p, j-k+p-n+2 m}^{(n)} u_{k-p+n, j}^{(2 m)}\right), \\
D_{2 n+1}^{+} u_{k, j}^{(2 m+1)}= & \sum_{p=1}^{k}\left((-1)^{p+1} u_{p+2 n+1, j}^{(2 n+1)} u_{k-p, j-p}^{(2 m+1)}+(-1)^{p(k-p)} u_{p+2 n+1, j-k+p+2 m+1}^{(2 n+1)} u_{k-p, j}^{(2 m+1)}\right), \\
D_{2 n}^{+} u_{k, j}^{(2 m+1)}= & \sum_{p=0}^{2 n}\left((-1)^{p} u_{p, j}^{(2 n)} u_{k-p+2 n, j-p+2 n}^{(2 m+1)}-(-1)^{p(k-p)} u_{p, j-k+p-2 n+2 m+1}^{(2 n)} u_{k-p+2 n, j}^{(2 m+1)}\right), \\
D_{n}^{-} u_{k, j}^{(m)}= & \sum_{p=0}^{n-1}\left((-1)^{(p+n) m} v_{p, j}^{(n)} u_{k+p-n, j+p-n}^{(m)}-(-1)^{(p+n)(k+p-n)} v_{p, j-k-p+n+m}^{(n)} u_{k+p-n, j}^{(m)}\right), \\
D_{n}^{+} v_{k, j}^{(m)}= & \sum_{p=0}^{n}\left((-1)^{(p+n) m} u_{p, j}^{(n)} v_{k+p-n, j-p+n}^{(m)}-(-1)^{(p+n)(k+p-n)} u_{p, j+k+p-n-m}^{(n)} v_{k+p-n, j}^{(m)}\right), \\
D_{n}^{-} v_{k, j}^{(2 m)}= & \sum_{p=0}^{n-1}\left(v_{p, j}^{(n)} v_{k-p+n, j+p-n}^{(2 m)}-(-1)^{(p+n)(k-p+n)} v_{p, j+k-p+n-2 m}^{(n)} v_{k-p+n, j}^{(2 m)}\right), \\
D_{2 n}^{-} v_{k, j}^{(2 m+1)}= & \sum_{p=0}^{2 n-1}\left((-1)^{p} v_{p, j}^{(2 n)} v_{k-p+2 n, j+p-2 n}^{(2 m+1)}-(-1)^{p(k-p)} v_{p, j+k-p+2 n-2 m-1}^{(2 n)} v_{k-p+2 n, j}^{(2 m+1)}\right), \\
D_{2 n+1}^{-} v_{k, j}^{(2 m+1)}= & \sum_{p=0}^{k}\left((-1)^{p+1} v_{p+2 n+1, j}^{(2 n+1)} v_{k-p, j+p}^{(2 m+1)}+(-1)^{p(k-p)} v_{p+2 n+1, j+k-p-2 m-1}^{(2 n+1)} v_{k-p, j}^{(2 m+1)}\right),
\end{aligned}
$$

where in the right-hand sides of these equations, all the fields $\left\{u_{k, j}^{(m)}, v_{k, j}^{(m)}\right\}$ with $k<0$ must be set equal to zero.

The $N=(2 \mid 2)$ supersymmetric 2D TL equation belongs to the system of (3.18), (3.19), (3.20), (3.21), (3.22), (3.23), (3.24), and (3.25). In order to see that, we consider (3.21) at $\{n=m=k=1\}$,

$$
D_{1}^{-} u_{1, j}=-v_{0, j}-v_{0, j+1},
$$

and (3.22) at $\{n=m=1, k=0\}$,

$$
D_{1}^{+} v_{0, j}=v_{0, j}\left(u_{1, j}-u_{1, j-1}\right) .
$$


Then, eliminating the field $u_{1, j}$ from (3.26) and (3.27), we obtain

$$
D_{1}^{+} D_{1}^{-} \ln v_{0, j}=v_{0, j+1}-v_{0, j-1} .
$$

Equation (3.28) reproduces the $N=(1 \mid 1)$ superfield form of the $N=(2 \mid 2)$ superconformal 2D TL equation (2.13) (see, e.g., $[5,6]$ and the references therein). Indeed, in terms of the superfield components

$$
g_{j} \equiv v_{0, j}\left|, \quad \gamma_{j}^{ \pm} \equiv\left(D_{1}^{ \pm} \ln v_{0, j}\right)\right|,
$$

where $g_{j}\left(\gamma_{j}^{ \pm}\right)$are the bosonic (fermionic) fields and $\mid$means the $t_{1}^{ \pm} \rightarrow 0$ limit, (3.28) coincides with (2.13) at $D_{2}^{-} \rightarrow-\partial_{1}$ and $D_{2}^{+} \rightarrow \partial_{2}$.

Now we define the supertrace of the operators $\mathbb{O}_{m}$,

$$
\mathbb{O}_{m}=\sum_{k=-\infty}^{\infty} f_{k, j}^{(m)} e^{(k-m) \partial}, \quad m \in \mathbb{Z},
$$

parameterized by the bosonic (fermionic) lattice functions $f_{2 k, j}^{(m)}\left(f_{2 k+1, j}^{(m)}\right)$, as a sum of all their diagonal elements of the trivial shift operator with $l=0\left(e^{0 \partial}=1\right)$ multiplied by the factor $(-1)^{j}$,

$$
\operatorname{str} \mathbb{O}=\sum_{j=-\infty}^{\infty}(-1)^{j} f_{m, j}^{(m)} .
$$

One can easily verify that the main property of supertraces

$$
\operatorname{str}\left[\mathbb{O}_{1}, \mathbb{O}_{2}\right\}=0
$$

is indeed satisfied for the case of the generalized graded bracket operation (3.13). Using this definition of the supertrace and Lax-pair representation (3.12), one can easily obtain conserved Hamiltonians of the $N=(1 \mid 1)$ 2D TL hierarchy

$$
H_{m}^{\alpha}=\operatorname{str}\left(L^{\alpha}\right)_{*}^{m}, \quad D_{n}^{ \pm} H_{m}^{\alpha}=0, \quad \alpha=+,-, m \in \mathbb{N} .
$$

From this formula it is obvious that all bosonic Hamiltonians corresponding to even values of $m$ are trivial $H_{2 n}^{\alpha}=0$ like a supertrace of the generalized graded bracket operation, while fermionic Hamiltonians at odd values of $m$ are not equal to zero $H_{2 n-1}^{\alpha} \neq 0$ in general. Using (3.14), we obtain more explicit superfield formulae for the latter,

$$
H_{2 n-1}^{+}=\sum_{j=-\infty}^{\infty}(-1)^{j} u_{2 n-1, j}^{(2 n-1)}, \quad H_{2 n-1}^{-}=\sum_{j=-\infty}^{\infty}(-1)^{j} v_{2 n-1, j}^{(2 n-1)},
$$

which in terms of superfield components look like

$$
\begin{aligned}
& s_{n}^{+}(u) \equiv H_{2 n-1}^{+}\left|=\sum_{j=-\infty}^{\infty}(-1)^{j} u_{2 n-1, j}^{(2 n-1)}\right|, \\
& s_{n}^{-}(v) \equiv H_{2 n-1}^{-}\left|=\sum_{j=-\infty}^{\infty}(-1)^{j} v_{2 n-1, j}^{(2 n-1)}\right| .
\end{aligned}
$$


The functionals $u_{m, j}^{(m)}$ and $v_{m, j}^{(m)}$ can be expressed in terms of the fields $v_{0, j}$ only (for details see [11]) and then using (3.19), (3.21), (3.24), and (3.4), in terms of the fields $\left(g_{j}, c_{j}, \gamma_{j}^{ \pm}\right)$ in such a way that $s_{m}^{-}(v)$ and $s_{m}^{+}(u)$ become fermionic integrals of motion for the $N=4$ TL equations (3.4).

To understand better how formulae (3.35) work, we finish this section with the examples and reproduce all fermionic Hamiltonians $S_{s, k}^{N=4}$ given by (3.1).

From (3.27) and the component correspondence (3.29), it directly follows that

$$
\begin{aligned}
s_{1}^{+}(u) & =\sum_{j=-\infty}^{\infty}(-1)^{j} u_{1, j}\left|=\frac{1}{2} \sum_{j=-\infty}^{\infty}(-1)^{j}\left(D_{1} \ln v_{0, j}\right)\right| \\
& =\frac{1}{2} \sum_{j=-\infty}^{\infty}(-1)^{j} \gamma_{j}^{+}=\frac{1}{2} S_{3,1}^{N=4},
\end{aligned}
$$

where $S_{3,1}^{N=4}$ is the fermionic integral in (3.1).

A more complicated problem is to obtain the next fermionic integral $s_{2}^{+}(u)$. First, using (3.12), one can find the explicit form of the functional $u_{3}^{(3)}$,

$$
s_{2}^{+}(u)=\sum_{j=-\infty}^{\infty}(-1)^{j} u_{3, j}^{(3)}\left|=3 \sum_{j=-\infty}^{\infty}(-1)^{j}\left(u_{3, j}+u_{2, j}\left(u_{1, j}-u_{1, j-1}\right)\right)\right| .
$$

Then, we consider (3.19) at $\{n=m=0, k=2\}$ and $\{n=m=0, k=1\}$,

$$
\begin{aligned}
& D_{1}^{+} u_{2, j}=u_{2, j}\left(u_{1, j-1}-u_{1, j}\right)-u_{3, j}+u_{3, j+1}, \\
& D_{1}^{+} u_{1, j}=u_{2, j}+u_{2, j+1},
\end{aligned}
$$

respectively. From (3.38), it follows that

$$
\sum_{j=-\infty}^{\infty}(-1)^{j} u_{3, j}=-\frac{1}{2} \sum_{j=-\infty}^{\infty}(-1)^{j}\left(D_{1}^{+} u_{2, j}+u_{2, j}\left(u_{1, j}-u_{1, j-1}\right)\right) ;
$$

the consequence of (3.39) and (3.27) is

$$
D_{1}^{+}\left(u_{1, j}-u_{1, j-1}\right)=u_{2, j+1}-u_{2, j-1}=\partial_{2}^{+} \ln v_{0, j} \longrightarrow u_{2, j}=\sum_{k=0}^{\infty} \partial \ln v_{0, j-2 k-1} ;
$$

and, at last, from (3.27) one can find that

$$
u_{1, j}=\sum_{k=0}^{\infty} D_{1}^{+} \ln v_{0, j-k} .
$$

Now it remains to substitute (3.40), (3.41), and (3.42) into (3.37) and to reproduce fermionic Hamiltonian $S_{3,2}^{N=4}=2 / 3 s_{2}^{+}(u)$ in (3.1) using (3.4) and (3.29).

Analogously, one can find that

$$
S_{1,1}^{N=4}=-2 s_{1}^{-}(v), \quad S_{1,2}^{N=4}=-\frac{2}{3} s_{2}^{-}(v) .
$$


The two remaining series of fermionic Hamiltonians in (3.1) can be easily derived from the obtained ones if one applies the automorphism transformations (3.10),

$$
\begin{aligned}
& S_{2, m}=S_{3, m}\left(\gamma_{j}^{+} \longrightarrow-(-1)^{j} \gamma_{j}^{-}, \gamma_{j}^{-} \longrightarrow(-1)^{j} \gamma_{j}^{+}\right), \\
& S_{4, m}=-S_{1, m}\left(\gamma_{j}^{+} \longrightarrow-(-1)^{j} \gamma_{j}^{-}, \gamma_{j}^{-} \longrightarrow(-1)^{j} \gamma_{j}^{+}\right) .
\end{aligned}
$$

3.3. Transition to the canonical basis for the $N=4 \mathrm{TL}$ equations. Our next task is to rewrite the $N=4 \mathrm{TL}$ equations (3.4) in a canonical basis where these equations admit a Lagrangian formulation that is important in connection with the quantization problem.

We introduce the new basis $\left\{x_{j}, p_{j}, \chi_{j}^{+}, \chi_{j}^{-}\right\}$in the phase space $\left\{g_{j}, c_{j}, \gamma_{j}^{+}, \gamma_{j}^{-}\right\}$:

$$
\begin{array}{ll}
g_{j}=i e^{x_{j}-x_{j-1}}, & \gamma_{j}^{+}=\chi_{j}^{-}+(-1)^{j} \chi_{j-1}^{+}, \\
c_{j}=-(-1)^{j} p_{j}, & \gamma_{j}^{-}=i\left(\chi_{j-1}^{-}-(-1)^{j} \chi_{j}^{+}\right),
\end{array}
$$

where $i$ is the imaginary unity and we suppose that the new fields go to zero at infinity:

$$
\lim _{j \rightarrow \pm \infty}\left\{x_{j}, p_{j}, \chi_{j}^{+}, \chi_{j}^{-}\right\}=0
$$

In terms of the new coordinates, the first Hamiltonian structure (3.2) becomes canonical,

$$
\left\{x_{i}, p_{j}\right\}_{1}=\delta_{i, j}, \quad\left\{\chi_{i}^{-}, \chi_{j}^{+}\right\}_{1}=\delta_{i, j}
$$

and the Hamiltonians in (3.1) take the following form:

$$
\begin{aligned}
H_{1} & =-\sum_{j=-\infty}^{\infty} p_{j}, \\
H_{2} & =\sum_{j=-\infty}^{\infty}(-1)^{j}\left(\frac{1}{2} p_{j}^{2}-e^{x_{j}-x_{j-2}}-e^{x_{j}-x_{j-1}}\left(\chi_{j-1}^{-}-(-1)^{j} \chi_{j}^{+}\right)\left(\chi_{j}^{-}+(-1)^{j} \chi_{j-1}^{+}\right)\right), \\
S_{1,1} & =i \sum_{j=-\infty}^{\infty}(-1)^{j}\left(\chi_{j-1}^{-}-(-1)^{j} \chi_{j}^{+}\right), \\
S_{2,1}= & i \sum_{j=-\infty}^{\infty}\left(\chi_{j-1}^{-}-(-1)^{j} \chi_{j}^{+}\right), \\
S_{3,1}= & \sum_{j=-\infty}^{\infty}(-1)^{j}\left(\chi_{j}^{-}+(-1)^{j} \chi_{j-1}^{+}\right), \\
S_{4,1}= & \sum_{j=-\infty}^{\infty}\left(\chi_{j}^{-}+(-1)^{j} \chi_{j-1}^{+}\right) .
\end{aligned}
$$


The Hamiltonian $\mathrm{H}_{2}$ generates the following equations via the first Hamiltonian structure (3.47):

$$
\begin{aligned}
& \partial x_{j}=-(-1)^{j} p_{j} \\
& \partial p_{j}=-(-1)^{j}\left(e^{x_{j}-x_{j-2}}-e^{x_{j+2}-x_{j}}+e^{x_{j}-x_{j-1}}\left(\chi_{j-1}^{-}-(-1)^{j} \chi_{j}^{+}\right)\left(\chi_{j}^{-}+(-1)^{j} \chi_{j-1}^{+}\right)\right. \\
& \left.+e^{x_{j+1}-x_{j}}\left(\chi_{j}^{-}+(-1)^{j} \chi_{j+1}^{+}\right)\left(\chi_{j+1}^{-}-(-1)^{j} \chi_{j}^{+}\right)\right), \\
& \partial \chi_{j}^{-}=-\left(e^{x_{j+1}-x_{j}}\left(\chi_{j}^{-}+(-1)^{j} \chi_{j+1}^{+}\right)+e^{x_{j}-x_{j-1}}\left(\chi_{j}^{-}+(-1)^{j} \chi_{j-1}^{+}\right)\right) \text {, } \\
& \partial \chi_{j}^{+}=-(-1)^{j}\left(e^{x_{j}-x_{j-1}}\left(\chi_{j-1}^{-}-(-1)^{j} \chi_{j}^{+}\right)+e^{x_{j+1}-x_{j}}\left(\chi_{j+1}^{-}-(-1)^{j} \chi_{j}^{+}\right)\right) .
\end{aligned}
$$

Following the standard procedure, one can derive the Lagrangian $\mathscr{L}$ and the action $\mathscr{T}$,

$$
\begin{aligned}
\mathscr{S}=\int d t \mathscr{L}=\int d t\left[\sum_{j=-\infty}^{\infty} p_{j} \frac{\partial}{\partial t} x_{j}+\chi_{j}^{-} \frac{\partial}{\partial t} \chi_{j}^{+}-H_{2}\right] \\
=\int d t \sum_{j=-\infty}^{\infty}\left[\frac{1}{2}\left(\frac{\partial}{\partial t} x_{j}\right)^{2}+\chi_{j}^{-} \frac{\partial}{\partial t} \chi_{j}^{+}\right. \\
\left.+(-1)^{j}\left(e^{x_{j}-x_{j-2}}+e^{x_{j}-x_{j-1}}\left(\chi_{j-1}^{-}-(-1)^{j} \chi_{j}^{+}\right)\left(\chi_{j}^{-}+(-1)^{j} \chi_{j-1}^{+}\right)\right)\right] .
\end{aligned}
$$

The variation of the action $\mathscr{S}$ with respect to the fields $\left\{x_{j}, \chi_{j}^{-}, \chi_{j}^{+}\right\}$produces the equations of motion (3.49) for them with reversed sign of the time $(\partial \rightarrow-\partial / \partial t)$ where the momenta $p_{j}$ are replaced by $(-1)^{j}(\partial / \partial t) x_{j}$.

One important remark is in order. There is no one-to-one correspondence between the phase space bases $\left\{g_{j}, c_{j}, \gamma_{j}^{+}, \gamma_{j}^{-}\right\}$and $\left\{x_{j}, p_{j}, \chi_{j}^{+}, \chi_{j}^{-}\right\}$in (3.45). Transformation (3.45) can rather be treated as a reduction of the primary phase space $\left\{g_{j}, c_{j}, \gamma_{j}^{+}, \gamma_{j}^{-}\right\}$to the subspace $\left\{x_{j}, p_{j}, \chi_{j}^{+}, \chi_{j}^{-}\right\}$with a smaller symmetry. Indeed, the direct consequence of (3.45) and (3.46) is the following constraints on the original fields:

$$
\prod_{k=-\infty}^{\infty}\left(-i g_{k}\right)=1, \quad \sum_{k=-\infty}^{\infty}\left(\gamma_{k}^{-}-i \gamma_{k}^{+}\right)=0, \quad \sum_{k=-\infty}^{\infty}(-1)^{k}\left(\gamma_{k}^{-}+i \gamma_{k}^{+}\right)=0 .
$$

Equations (3.51) restrict the phase space of (3.4) and change the symmetry properties of the latter. The first manifestation of such a restriction is the fact that Hamiltonians $H_{1}$ and $S_{n, 1}$ in (3.48) no longer belong to the center of the first Hamiltonian structure and generate the following nontrivial flows via the algebra (3.47):

$$
\begin{aligned}
& \partial_{H_{1}} x_{j}=1, \quad D_{S_{1,1}} \chi_{j}^{-}=-i, \quad D_{S_{1,1}} \chi_{j}^{+}=-(-1)^{j} i, \\
& D_{S_{2,1}} \chi_{j}^{-}=-(-1)^{j} i, \quad D_{S_{2,1}} \chi_{j}^{+}=i, \quad D_{S_{3,1}} \chi_{j}^{-}=1, \\
& D_{S_{3,1}} \chi_{j}^{+}=(-1)^{j}, \quad D_{S_{4,1}} \chi_{j}^{-}=-(-1)^{j}, \quad D_{S_{4,1}} \chi_{j}^{+}=1 \text {. }
\end{aligned}
$$

Furthermore, conditions (3.51) break the $N=4$ supersymmetry transformations (3.4), (3.5), and (3.6) and in order to restore the $N=4$ supersymmetry, one needs to impose 
the following additional constraints on the fields:

$$
\sum_{k=-\infty}^{\infty}(-1)^{k} \gamma_{k}^{ \pm}=0, \quad \sum_{k=-\infty}^{\infty} \gamma_{k}^{ \pm}=0, \quad \sum_{k=-\infty}^{\infty}(-1)^{k} c_{k}=0 .
$$

However, the following $N=2$ supersymmetry transformations in terms of fermionic flows (3.5) and (3.6):

$$
\tilde{D}_{1}=i D_{1}+D_{3}, \quad \tilde{D}_{2}=i D_{2}-D_{4}, \quad \tilde{D}_{1}^{2}=2 \partial, \quad \tilde{D}_{2}^{2}=-2 \partial
$$

are consistent with the constraints (3.51) and provide the $N=2$ supersymmetry for the infinite TL in the canonical basis (3.49),

$$
\begin{aligned}
& \tilde{D}_{1} x_{j}=\chi_{j}^{-}+(-1)^{j} \chi_{j}^{+}, \\
& \tilde{D}_{1} p_{j}=e^{x_{j+1}-x_{j}}\left(\chi_{j+1}^{+}-\chi_{j}^{+}+(-1)^{j}\left(\chi_{j}^{-}+\chi_{j+1}^{-}\right)\right)+e^{x_{j}-x_{j-1}}\left(\chi_{j-1}^{+}-\chi_{j}^{+}+(-1)^{j}\left(\chi_{j}^{-}+\chi_{j-1}^{-}\right)\right), \\
& \tilde{D}_{1} \chi_{j}^{-}=e^{x_{j+1}-x_{j}}-e^{x_{j}-x_{j-1}}-(-1)^{j} p_{j}, \\
& \tilde{D}_{1} \chi_{j}^{+}=(-1)^{j}\left(e^{x_{j}-x_{j-1}}-e^{x_{j+1}-x_{j}}\right)-p_{j}, \\
& \tilde{D}_{2} x_{j}=\chi_{j}^{+}-(-1)^{j} \chi_{j}^{-}, \\
& \tilde{D}_{2} p_{j}=e^{x_{j+1}-x_{j}}\left(\chi_{j+1}^{-}-\chi_{j}^{-}-(-1)^{j}\left(\chi_{j}^{+}+\chi_{j+1}^{+}\right)\right)+e^{x_{j}-x_{j-1}}\left(\chi_{j-1}^{-}-\chi_{j}^{-}-(-1)^{j}\left(\chi_{j}^{+}+\chi_{j-1}^{+}\right)\right), \\
& \tilde{D}_{2} \chi_{j}^{+}=e^{x_{j+1}-x_{j}}-e^{x_{j}-x_{j-1}}+(-1)^{j} p_{j}, \\
& \tilde{D}_{2} \chi_{j}^{-}=(-1)^{j}\left(e^{x_{j+1}-x_{j}}-e^{x_{j}-x_{j-1}}\right)-p_{j} .
\end{aligned}
$$

Equation (3.49) can be represented in the superfield form

$$
\mathscr{D}_{+} \mathscr{D}_{-} \Phi_{j}=2(-1)^{j}\left(e^{\Phi_{j+1}-\Phi_{j}}-e^{\Phi_{j}-\Phi_{j-1}}\right),
$$

where $\Phi_{j}$ is the bosonic $N=2$ superfield with the components

$$
x_{j}=\Phi_{j}\left|, \quad \chi_{j}^{-}+(-1)^{j} \chi_{j}^{+}=\mathscr{D}_{+} \Phi_{j}\right|, \quad \chi_{j}^{+}-(-1)^{j} \chi_{j}^{-}=\mathscr{D}_{-} \Phi_{j} \mid .
$$

Here, $\mid$ means the $\theta^{ \pm} \rightarrow 0$ limit and $\mathscr{D}_{ \pm}$are the fermionic covariant derivatives

$$
\mathscr{D}_{ \pm}=\frac{\partial}{\partial_{\theta^{ \pm}}} \pm 2 \theta^{ \pm} \partial, \quad \mathscr{D}_{ \pm}^{2}= \pm 2 \partial, \quad\left\{\mathscr{D}_{+}, \mathscr{D}_{-}\right\}=0
$$


In order to rewrite the second Hamiltonian structure (3.3) in terms of the new fields $\left\{x_{j}, p_{j}, \chi_{j}^{+}, \chi_{j}^{-}\right\}$, we invert (3.45),

$$
\begin{aligned}
& x_{j}=c \sum_{k=-\infty}^{j}\left(\ln g_{k}-\frac{i \pi}{2}\right)+(c-1) \sum_{k=j+1}^{\infty}\left(\ln g_{k}-\frac{i \pi}{2}\right), \\
& p_{j}=-(-1)^{j} c_{j}, \\
& \chi_{j}^{+}=(-1)^{j} \sum_{k=-\infty}^{-1}\left(c_{+} \gamma_{j+2 k+1}^{+}+i c_{-} \gamma_{j+2 k+2}^{-}\right)+(-1)^{j} \sum_{0}^{\infty}\left(\left(c_{+}-1\right) \gamma_{j+2 k+1}^{+}+i\left(c_{-}-1\right) \gamma_{j+2 k+2}^{-}\right), \\
& \chi_{j}^{-}=\sum_{k=-\infty}^{-1}\left(c_{+} \gamma_{j+2 k+2}^{+}+i c_{-} \gamma_{j+2 k+1}^{-}\right)-\sum_{0}^{\infty}\left(\left(c_{+}-1\right) \gamma_{j+2 k+1}^{+}+i\left(c_{-}-1\right) \gamma_{j+2 k+1}^{-}\right),
\end{aligned}
$$

and find the Poisson brackets between the fields $\left\{x_{j}, p_{j}, \chi_{j}^{+}, \chi_{j}^{-}\right\}$using relations (3.3). Equations (3.59) contain three arbitrary parameters $c$ and $c_{ \pm}$. However, the second Hamiltonian structure (3.3) is not consistent with constraints (3.51) in general, and it is not guaranteed a priori that the Poisson brackets obtained in such a way obey the Jacobi identities. The test of the Jacobi identities shows that the Poisson brackets obtained form a closed algebra only at $c=1$ and $c_{ \pm}=0$ and have the following explicit form:

$$
\begin{aligned}
\left\{x_{i}, x_{j}\right\}_{2}= & (-1)^{j} \delta_{i, j}^{+}-(-1)^{i} \delta_{i, j}^{-}, \\
\left\{x_{i}, p_{j}\right\}_{2}= & -(-1)^{j} p_{j} \delta_{i, j}, \\
\left\{p_{i}, p_{j}\right\}_{2}= & -(-1)^{j}\left(e^{x_{i}-x_{j}}\left(\chi_{i}^{-}+(-1)^{i} \chi_{i-1}^{+}\right)\left(\chi_{i-1}^{-}-(-1)^{i} \chi_{i}^{+}\right) \delta_{i, j+1}+e^{x_{i}-x_{j}} \delta_{i, j+2}\right. \\
& \left.+e^{x_{j}-x_{i}}\left(\chi_{j}^{-}+(-1)^{j} \chi_{j-1}^{+}\right)\left(\chi_{j-1}^{-}-(-1)^{j} \chi_{j}^{+}\right) \delta_{i, j-1}-e^{x_{j}-x_{i}} \delta_{i, j-2}\right), \\
\left\{p_{i}, \chi_{j}^{ \pm}\right\}_{2}= & \varrho_{i+j}^{+}\left[e^{x_{i+1}-x_{i}}\left(\mp \chi_{i}^{ \pm}+(-1)^{i} \chi_{i+1}^{\mp}\right) \delta_{i, j-1}^{+}+e^{x_{i}-x_{i-1}}\left( \pm \chi_{i}^{ \pm}-(-1)^{i} \chi_{i-1}^{\mp}\right) \delta_{i, j+1}^{+}\right] \\
& +\varrho_{i+j}^{-}\left[e^{x_{i}-x_{i-1}}\left( \pm \chi_{i-1}^{ \pm}+(-1)^{i} \chi_{i}^{\mp}\right) \delta_{i, j+1}^{+}+e^{x_{i+1}-x_{i}}\left(\mp \chi_{i+1}^{ \pm}-(-1)^{i} \chi_{i}^{\mp}\right) \delta_{i, j-2}^{+}\right], \\
\left\{x_{i}, \chi_{j}^{ \pm}\right\}_{2}= & \varrho_{i+j}^{+}\left[(-1)^{j}\left(\chi_{j}^{ \pm}-\chi_{i}^{ \pm}\right)+2 \sum_{k=1}^{(i-j) / 2}\left(\mp \chi_{i-2 k+1}^{ \pm} \pm(-1)^{j} \chi_{j+2 k}^{ \pm}\right)\right] \delta_{i, j+1}^{+} \\
& +\varrho_{i+j}^{-}\left[\left(\mp \chi_{i}^{\mp}+(-1)^{j} \chi_{j}^{ \pm}\right) \delta_{i, j}^{+}+2 \sum_{k=1}^{(i-j-1) / 2}\left(\mp \chi_{i-2 k}^{\mp}+(-1)^{j} \chi_{j+2 k}^{ \pm}\right) \delta_{i, j+1}^{+}\right], \\
\left\{\chi_{i}^{-}, \chi_{j}^{+}\right\}_{2}= & \varrho_{i+j}^{+}\left[2(-1)^{j} \delta_{i, j} \sum_{k=i+1}^{\infty} p_{k}+\left(e^{x_{i}-x_{i-1}}+e^{x_{i+1}-x_{i}}-(-1)^{j}\left(p_{i}+2 \sum_{k=i+1}^{\infty} p_{k}\right)\right) \delta_{i, j}^{+}\right. \\
& \left.+\left(e^{x_{j}-x_{j-1}}+e^{x_{j+1}-x_{j}}+(-1)^{j}\left(p_{j}+2 \sum_{k=j+1}^{\infty} p_{k}\right)\right) \delta_{i, j}^{-}\right],
\end{aligned}
$$




$$
\begin{aligned}
\left\{\chi_{i}^{ \pm}, \chi_{j}^{ \pm}\right\}_{2}=\varrho_{i+j}^{-}[ & \left(-(-1)^{i} e^{x_{i+1}-x_{i}} \mp p_{i} \mp 2 \sum_{k=i+1}^{\infty} p_{k}\right) \delta_{i, j}^{+}-(-1)^{i} e^{x_{i}-x_{i-1}} \delta_{i, j+1}^{+} \\
& \left.+\left(-(-1)^{j} e^{x_{j+1}-x_{j}} \mp p_{j} \mp 2 \sum_{k=j+1}^{\infty} p_{k}\right) \delta_{i, j}^{-}-(-1)^{j} e^{x_{j}-x_{j-1}} \delta_{i, j-1}^{-}\right],
\end{aligned}
$$

where we have introduced the notation

$$
\varrho_{j}^{ \pm}=\frac{\left(1 \pm(-1)^{j}\right)}{2}, \quad \delta_{i, j}^{+}=\left\{\begin{array}{ll}
1, & \text { if } i>j, \\
0, & \text { if } i \leq j,
\end{array} \quad \delta_{i, j}^{-}= \begin{cases}1, & \text { if } i<j, \\
0, & \text { if } i \geq j,\end{cases}\right.
$$

with the property

$$
\delta_{i, j}^{-}+\delta_{i, j}^{+}+\delta_{i, j} \equiv 1
$$

One can check that the Hamiltonian $H_{1}$ in (3.48) reproduces (3.49) via the second Hamiltonian structure (3.60).

\section{Reduction: $1 \mathrm{D} N=2$ supersymmetric TL hierarchy}

4.1. Bi-Hamiltonian structure of the $1 \mathrm{D} N=2 \mathrm{TL}$ hierarchy. The $1 \mathrm{D} N=2$ TL hierarchy was proposed and studied in detail in [3]. In this section, we reproduce its biHamiltonian description [3] reducing the bi-Hamiltonian structure (2.28) and (2.29) of the $1 \mathrm{D}$ generalized fermionic TL hierarchy by reduction constraint (2.21).

Our starting point is (2.16) with boundary conditions (2.4a) and (2.4c) in 1D space (2.22). Substituting reduction constraint (2.21) into (2.16), we obtain the following equation for the fields $\bar{a}_{j}$ :

$$
\partial \bar{a}_{j}=\beta_{j} \bar{\alpha}_{j}+\alpha_{j} \bar{\beta}_{j}
$$

which can easily be solved. Here, we note that the system $(2.16)$ is scale-invariant and length dimensions of the involved fields are $\left[b_{j}\right]=\left[\bar{b}_{j}\right]=-2,\left[a_{j}\right]=\left[\bar{a}_{j}\right]=-1,\left[\beta_{j}\right]=$ $\left[\bar{\beta}_{j}\right]=-3 / 2$, and $\left[\alpha_{j}\right]=\left[\bar{\alpha}_{j}\right]=-1 / 2$. Keeping this in mind, we obtain the scale-invariant solution to $(4.1)$,

$$
\bar{a}_{j}=-\frac{\beta_{j} \bar{\beta}_{j}}{b_{j}} .
$$

Substituting this solution into (2.16) at $\partial_{2}=\partial_{1}=\partial$, we obtain the following equations:

$$
\begin{gathered}
\partial b_{j}=b_{j}\left(a_{j}-a_{j-1}\right), \quad \partial a_{j}=b_{j+1}-b_{j}+\beta_{j} \bar{\alpha}_{j}+\alpha_{j+1} \bar{\beta}_{j+1}, \\
\partial \beta_{j}=a_{j} \beta_{j}-b_{j} \alpha_{j}, \quad \partial \bar{\beta}_{j}=-a_{j-1} \bar{\beta}_{j}-b_{j} \bar{\alpha}_{j}, \\
\partial \alpha_{j}=\beta_{j}-\beta_{j-1}, \quad \partial \bar{\alpha}_{j}=\bar{\beta}_{j}-\bar{\beta}_{j+1} .
\end{gathered}
$$

Fermionic symmetries (2.17) become inconsistent after reduction $\bar{b}_{j}=0$ because in this case the fields $e_{j}$ in (2.19) become singular. As concerns fermionic symmetries in (2.18), 
they are consistent and take the following form:

$$
\begin{array}{ll}
D_{1} b_{j}=b_{j}\left(\alpha_{j}-\bar{\alpha}_{j}\right), & D_{2} b_{j}=b_{j}\left(\alpha_{j}+\bar{\alpha}_{j}\right), \\
D_{1} a_{j}=\bar{\beta}_{j+1}+\beta_{j}, & D_{2} a_{j}=-\bar{\beta}_{j+1}+\beta_{j}, \\
D_{1} \beta_{j}=-b_{j}+\beta_{j} \bar{\alpha}_{j}, & D_{2} \beta_{j}=b_{j}-\beta_{j} \bar{\alpha}_{j}, \\
D_{1} \bar{\beta}_{j}=b_{j}-\bar{\beta}_{j} \alpha_{j}, & D_{2} \bar{\beta}_{j}=b_{j}-\bar{\beta}_{j} \alpha_{j}, \\
D_{1} \alpha_{j}=-a_{j-1}-\frac{\beta_{j} \bar{\beta}_{j}}{b_{j}}, & D_{2} \alpha_{j}=a_{j-1}+\frac{\beta_{j} \bar{\beta}_{j}}{b_{j}}, \\
D_{1} \bar{\alpha}_{j}=-a_{j}-\frac{\beta_{j} \bar{\beta}_{j}}{b_{j}}, & D_{2} \bar{\alpha}_{j}=-a_{j}-\frac{\beta_{j} \bar{\beta}_{j}}{b_{j}} .
\end{array}
$$

The system (4.3) is supplied with the boundary conditions (2.4a) and (2.4c). We recall that for the boundary conditions (2.4c), the system (2.16) does not possess any supersymmetry (see the paragraph with (2.11)). In terms of fields (2.15), the boundary conditions $(2.4 \mathrm{a})$ and $(2.4 \mathrm{c})$ are

$$
\begin{gathered}
\lim _{j \rightarrow \pm \infty}\left\{b_{j}, a_{j}, \alpha_{j}, \bar{\alpha}_{j}, \beta_{j}, \bar{\beta}_{j}\right\}=0, \\
\lim _{j \rightarrow \pm \infty} b_{j}=1, \quad \lim _{j \rightarrow \pm \infty}\left\{a_{j}, \alpha_{j}, \bar{\alpha}_{j}, \beta_{j}, \bar{\beta}_{j}\right\}=0,
\end{gathered}
$$

respectively. Therefore, we conclude that system (4.3) possesses $N=2$ supersymmetry only for the boundary conditions (4.5a), while for the boundary conditions (4.5b), it is not supersymmetric.

The first (2.28) and second (2.29) Hamiltonian structures in the basis $\left\{b_{j}, \bar{b}_{j}, a_{j}, \bar{a}_{j}, \alpha_{j}\right.$, $\left.\bar{\alpha}_{j}, \beta_{j}, \bar{\beta}_{j}\right\}$ in (2.15) look like

$$
\begin{array}{ll}
\left\{b_{i}, a_{j}\right\}_{1}=b_{i}\left(\delta_{i, j}-\delta_{i, j+1}\right), & \left\{\bar{b}_{i}, \bar{a}_{j}\right\}_{1}=\bar{b}_{i}\left(\delta_{i, j+1}-\delta_{i, j}\right), \\
\left\{a_{i}, \beta_{j}\right\}_{1}=-\beta_{j} \delta_{i, j}, & \left\{\bar{a}_{i}, \bar{\beta}_{j}\right\}_{1}=\bar{\beta}_{j} \delta_{i, j}, \\
\left\{\bar{a}_{i}, \beta_{j}\right\}_{1}=-\beta_{j} \delta_{i, j}, & \left\{a_{i}, \bar{\beta}_{j}\right\}_{1}=\bar{\beta}_{j} \delta_{i, j-1}, \\
\left\{\beta_{i}, \bar{\beta}_{j}\right\}_{1}=b_{j} \delta_{i, j}-\bar{b}_{j} \delta_{i, j-1}, & \left\{\alpha_{i}, \bar{\alpha}_{j}\right\}_{1}=\delta_{i, j}-\delta_{i, j+1}, \\
\left\{b_{i}, b_{j}\right\}_{2}=-b_{i} b_{j}\left(\delta_{i, j+1}-\delta_{i, j-1}\right), & \left\{\bar{b}_{i}, \bar{b}_{j}\right\}_{2}=\bar{b}_{i} \bar{b}_{j}\left(\delta_{i, j+1}-\delta_{i, j-1}\right), \\
\left\{a_{i}, a_{j}\right\}_{2}=-\left(b_{i} \delta_{i, j+1}-b_{j} \delta_{i, j-1}\right), & \left\{\bar{a}_{i}, \bar{a}_{j}\right\}_{2}=\bar{b}_{i} \delta_{i, j+1}-\bar{b}_{j} \delta_{i, j-1}, \\
\left\{a_{i}, \bar{a}_{j}\right\}_{2}=\bar{\alpha}_{j} \beta_{i} \delta_{i, j}-\alpha_{j} \bar{\beta}_{j} \delta_{i, j-1}, & \left\{b_{i}, a_{j}\right\}_{2}=-b_{i} a_{j}\left(\delta_{i, j+1}-\delta_{i, j}\right), \\
\left\{\bar{b}_{i}, \bar{a}_{j}\right\}_{2}=\bar{b}_{i} \bar{a}_{j}\left(\delta_{i, j+1}-\delta_{i, j}\right), & \left\{b_{i}, \alpha_{j}\right\}_{2}=-b_{i} \alpha_{j} \delta_{i, j}, \\
\left\{\bar{b}_{i}, \bar{\alpha}_{j}\right\}_{2}=\bar{b}_{i} \bar{\alpha}_{j} \delta_{i, j+1}, & \left\{b_{i}, \bar{\alpha}_{j}\right\}_{2}=b_{i} \bar{\alpha}_{j} \delta_{i, j}, \\
\left\{\bar{b}_{i}, \alpha_{j}\right\}_{2}=-\bar{b}_{i} \alpha_{j} \delta_{i, j}, & \left\{b_{i}, \beta_{j}\right\}_{2}=-b_{i} \beta_{j} \delta_{i, j+1}, \\
\left\{\bar{b}_{i}, \bar{\beta}_{j}\right\}_{2}=\bar{b}_{i} \bar{\beta}_{j} \delta_{i, j+1}, & \left\{\bar{b}_{i}, \beta_{j}\right\}_{2}=-\bar{b}_{i} \beta_{j} \delta_{i, j}, \\
\left\{b_{i}, \bar{\beta}_{j}\right\}_{2}=b_{i} \bar{\beta}_{j} \delta_{i, j-1}, & \left\{a_{i}, \beta_{j}\right\}_{2}=-a_{i} \beta_{j} \delta_{i, j}+b_{j} \alpha_{j} \delta_{i, j-1}, \\
\left\{\bar{a}_{i}, \bar{\beta}_{j}\right\}_{2}=\bar{a}_{i} \bar{\beta}_{j} \delta_{i, j}+\bar{b}_{j} \bar{\alpha}_{i} \delta_{i, j-1}, & \left\{a_{i}, \bar{\beta}_{j}\right\}_{2}=a_{i} \bar{\beta}_{j} \delta_{i, j-1}+b_{i} \bar{\alpha}_{j} \delta_{i, j},
\end{array}
$$




$$
\begin{array}{ll}
\left\{\bar{a}_{i}, \beta_{j}\right\}_{2}=-\bar{a}_{i} \beta_{j} \delta_{i, j}+\bar{b}_{i} \alpha_{i} \delta_{i, j+1}, & \left\{\bar{\beta}_{i}, \alpha_{j}\right\}_{2}=-\left(\bar{\beta}_{i} \alpha_{j} \delta_{i, j}+\bar{b}_{i} \delta_{i, j+1}-b_{i} \delta_{i, j-1}\right), \\
\left\{\bar{a}_{i}, \bar{\alpha}_{j}\right\}_{2}=-\bar{\beta}_{i} \delta_{i, j+1}, & \left\{\alpha_{i}, \bar{\alpha}_{j}\right\}_{2}=-a_{j} \delta_{i, j+1}+\bar{a}_{j} \delta_{i, j}, \\
\left\{\bar{a}_{i}, \alpha_{j}\right\}_{2}=-\beta_{i} \delta_{i, j-1}, & \left\{\beta_{i}, \bar{\alpha}_{j}\right\}_{2}=\beta_{i} \bar{\alpha}_{j} \delta_{i, j}-b_{i} \delta_{i, j+1}+\bar{b}_{j} \delta_{i, j-1}, \\
\left\{a_{i}, \alpha_{j}\right\}_{2}=-\beta_{i} \delta_{i, j}, & \left\{\bar{\beta}_{i}, \beta_{j}\right\}_{2}=\left(\beta_{i} \bar{\beta}_{j}-\bar{b}_{j} a_{i}\right) \delta_{i, j-1}+\left(\beta_{i} \bar{\beta}_{j}+b_{i} \bar{a}_{j}\right) \delta_{i, j}, \\
\left\{a_{i}, \bar{\alpha}_{j}\right\}_{2}=-\bar{\beta}_{j} \delta_{i, j-1}, &
\end{array}
$$

respectively. One can easily see that the algebras in (4.6) are consistent with the reduction constraints (2.21) and (4.2), so the $1 \mathrm{D} N=2$ supersymmetric TL equations (4.3) can be represented as a bi-Hamiltonian system with the first Hamiltonian structure:

$$
\begin{gathered}
\left\{b_{i}, a_{j}\right\}_{1}=b_{i}\left(\delta_{i, j}-\delta_{i, j+1}\right), \quad\left\{a_{i}, \beta_{j}\right\}_{1}=-\beta_{j} \delta_{i, j}, \quad\left\{a_{i}, \bar{\beta}_{j}\right\}_{1}=\bar{\beta}_{j} \delta_{i, j-1}, \\
\left\{\beta_{i}, \bar{\beta}_{j}\right\}_{1}=b_{j} \delta_{i, j}, \quad\left\{\alpha_{i}, \bar{\alpha}_{j}\right\}_{1}=\delta_{i, j}-\delta_{i, j+1},
\end{gathered}
$$

and the second Hamiltonian structure:

$$
\begin{array}{ll}
\left\{b_{i}, b_{j}\right\}_{2}=-b_{i} b_{j}\left(\delta_{i, j+1}-\delta_{i, j-1}\right), & \left\{b_{i}, a_{j}\right\}_{2}=-b_{i} a_{j}\left(\delta_{i, j+1}-\delta_{i, j}\right), \\
\left\{a_{i}, a_{j}\right\}_{2}=-b_{i} \delta_{i, j+1}+b_{j} \delta_{i, j-1}, & \left\{b_{i}, \alpha_{j}\right\}_{2}=-b_{i} \alpha_{j} \delta_{i, j}, \\
\left\{b_{i}, \bar{\alpha}_{j}\right\}_{2}=b_{i} \bar{\alpha}_{j} \delta_{i, j}, & \left\{b_{i}, \beta_{j}\right\}_{2}=-b_{i} \beta_{j} \delta_{i, j+1}, \\
\left\{b_{i}, \bar{\beta}_{j}\right\}_{2}=b_{i} \bar{\beta}_{j} \delta_{i, j-1}, & \left\{a_{i}, \beta_{j}\right\}_{2}=-a_{i} \beta_{j} \delta_{i, j}+b_{j} \alpha_{j} \delta_{i, j-1}, \\
\left\{a_{i}, \bar{\beta}_{j}\right\}_{2}=a_{i} \bar{\beta}_{j} \delta_{i, j-1}+b_{i} \bar{\alpha}_{j} \delta_{i, j}, & \left\{a_{i}, \alpha_{j}\right\}_{2}=-\beta_{i} \delta_{i, j}, \\
\left\{a_{i}, \bar{\alpha}_{j}\right\}_{2}=-\bar{\beta}_{j} \delta_{i, j-1}, & \left\{\bar{\beta}_{i}, \alpha_{j}\right\}_{2}=-\bar{\beta}_{i} \alpha_{j} \delta_{i, j}+b_{i} \delta_{i, j-1}, \\
\left\{\beta_{i}, \bar{\alpha}_{j}\right\}_{2}=\beta_{i} \bar{\alpha}_{j} \delta_{i, j}-b_{i} \delta_{i, j+1}, & \left\{\beta_{i}, \bar{\beta}_{j}\right\}_{2}=\beta_{i} \bar{\beta}_{j} \delta_{i, j-1}, \\
\left\{\alpha_{i}, \bar{\alpha}_{j}\right\}_{2}=-a_{j} \delta_{i, j+1}-\frac{\beta_{j} \bar{\beta}_{j}}{b_{j}} \delta_{i, j}, &
\end{array}
$$

where when calculating we have substituted the reduction constraints (2.21) and (4.2) into the original algebras (4.6). We also present a few first bosonic and fermionic Hamiltonians of the $1 \mathrm{D} N=2$ supersymmetric TL hierarchy obtained from Hamiltonians (2.25) and (2.35) using reduction constraints (2.21) and (4.2):

$$
\begin{aligned}
H_{1}^{N=2} & =-\sum_{j=-\infty}^{\infty}\left(a_{j}+\frac{\beta_{j} \bar{\beta}_{j}}{b_{j}}\right), \\
H_{2}^{N=2} & =-\sum_{j=-\infty}^{\infty}\left(\frac{1}{2} a_{j}^{2}+b_{j}+\alpha_{j} \bar{\beta}_{j}-\beta_{j} \bar{\alpha}_{j}\right), \\
S_{1,1}^{N=2} & =\sum_{j=-\infty}^{\infty}\left(\alpha_{j}+\bar{\alpha}_{j}\right), \\
S_{2,1}^{N=2} & =\sum_{j=-\infty}^{\infty}\left(\alpha_{j}-\bar{\alpha}_{j}\right),
\end{aligned}
$$




$$
\begin{aligned}
S_{1,2}^{N=2} & =\sum_{j=-\infty}^{\infty}\left(\beta_{j}-\bar{\beta}_{j}-\bar{\alpha}_{j} \frac{\beta_{j} \bar{\beta}_{j}}{b_{j}}+\left(\alpha_{j}-\bar{\alpha}_{j}\right) \sum_{k=-\infty}^{j-1}\left(a_{k}+\frac{\beta_{k} \bar{\beta}_{k}}{b_{k}}\right)\right), \\
S_{2,2}^{N=2} & =\sum_{j=-\infty}^{\infty}\left(\beta_{j}+\bar{\beta}_{j}+\bar{\alpha}_{j} \frac{\beta_{j} \bar{\beta}_{j}}{b_{j}}+\left(\alpha_{j}+\bar{\alpha}_{j}\right) \sum_{k=-\infty}^{j-1}\left(a_{k}+\frac{\beta_{k} \bar{\beta}_{k}}{b_{k}}\right)\right) .
\end{aligned}
$$

4.2. Transition to the canonical basis for the $1 \mathrm{D} N=2 \mathrm{TL}$ equations. The transition to the canonical basis for the $1 \mathrm{D} N=2$ supersymmetric TL equations (4.3) with nonperiodic boundary conditions is possible only for the boundary conditions (4.5b). As we have already mentioned, in this case the system (4.3) is not supersymmetric, nevertheless it can serve as a basement for the building of the periodic $N=2$ TL equations in the canonical basis. In this section, we briefly discuss the representation of the system (4.3) in the canonical basis.

Following [3], we introduce the new basis $\left\{x_{j}, p_{j}, \xi_{j}, \bar{\xi}_{j}, \eta_{j}, \bar{\eta}_{j}\right\}$ in the phase space $\left\{a_{j}\right.$, $\left.b_{j}, \alpha_{j}, \bar{\alpha}_{j}, \beta_{j}, \bar{\beta}_{j}\right\}$ :

$$
\begin{aligned}
& a_{i}=p_{i}, \quad b_{i}=e^{x_{i}-x_{i-1}}, \quad \beta_{i}=e^{x_{i}} \xi_{i}, \\
& \bar{\beta}_{i}=e^{-x_{i-1}} \bar{\xi}_{i}, \quad \bar{\alpha}_{i}=-\bar{\eta}_{i}, \quad \alpha_{i}=\eta_{i-1}-\eta_{i},
\end{aligned}
$$

with the zero boundary conditions at infinity

$$
\lim _{j \rightarrow \pm \infty}\left\{x_{j}, p_{j}, \xi_{j}, \bar{\xi}_{j}, \eta_{j}, \bar{\eta}_{j}\right\}=0
$$

In this basis the first Hamiltonian structure becomes canonical:

$$
\left\{x_{i}, p_{j}\right\}_{1}=\delta_{i, j}, \quad\left\{\xi_{i}, \bar{\xi}_{j}\right\}_{1}=\delta_{i, j}, \quad\left\{\eta_{i}, \bar{\eta}_{j}\right\}_{1}=\delta_{i, j}
$$

while the second Hamiltonian structure takes a more complicated form:

$$
\begin{array}{ll}
\left\{x_{i}, x_{j}\right\}_{2}=\delta_{i, j}^{-}-\delta_{i, j}^{+}, & \left\{x_{i}, p_{j}\right\}_{2}=p_{j} \delta_{i, j}, \\
\left\{p_{i}, p_{j}\right\}_{2}=e^{x_{j}-x_{i}} \delta_{i, j-1}-e^{x_{i}-x_{j}} \delta_{i, j+1}, & \left\{p_{i}, \xi_{j}\right\}_{2}=e^{-x_{i}}\left(\eta_{i}-\eta_{j}\right) \delta_{i, j-1}, \\
\left\{p_{i}, \eta_{j}\right\}_{2}=e^{x_{i}} \xi_{i}\left(\tilde{c}-\delta_{i, j}^{+}\right), & \left\{x_{i}, \xi_{j}\right\}_{2}=\xi_{j}\left(1-c-\delta_{i, j}^{-}\right), \\
\left\{x_{i}, \eta_{j}\right\}_{2}=\eta_{i}\left(1-\tilde{c}-\delta_{i, j}^{-}\right)+\eta_{j}\left(\delta_{i, j}^{-}-c\right), & \left\{\xi_{i}, \eta_{j}\right\}_{2}=\xi_{i} \eta_{i}\left(\tilde{c}-\delta_{i, j}^{+}\right)+\xi_{i} \eta_{j}\left(c-1+\delta_{i, j}^{+}\right), \\
\left\{p_{i}, \bar{\eta}_{j}\right\}_{2}=e^{-x_{i}} \bar{\xi}_{j} \delta_{i, j-1}, & \left\{p_{i}, \bar{\xi}_{j}\right\}_{2}=-e^{x_{i}} \bar{\eta}_{j} \delta_{i, j}, \\
\left\{x_{i}, \bar{\eta}_{j}\right\}_{2}=\bar{\eta}_{j}\left(c-\delta_{i, j}^{-}\right), & \left\{x_{i}, \bar{\xi}_{j}\right\}_{2}=\bar{\xi}_{j}\left(c-1+\delta_{i, j}^{-}\right), \\
\left\{\bar{\xi}_{i}, \bar{\eta}_{j}\right\}_{2}=\bar{\xi}_{i} \bar{\eta}_{j}\left(c-1+\delta_{i, j}^{+}\right), & \left\{\xi_{i}, \bar{\eta}_{j}\right\}_{2}=\xi_{i} \bar{\eta}_{j}\left(1-c-\delta_{i, j}^{+}\right)+e^{-x_{j}} \delta_{i, j+1}, \\
\left\{\eta_{i}, \bar{\eta}_{j}\right\}_{2}=\xi_{j} \bar{\xi}_{j}\left(\delta_{i, j}^{-}-\tilde{c}\right)+p_{j}\left(1-\tilde{c}-\delta_{i, j}^{+}\right), \\
\left\{\bar{\xi}_{i}, \eta_{j}\right\}_{2}=\left(\bar{\xi}_{i} \eta_{i}+e^{x_{i}}\right)\left(1-\tilde{c}-\delta_{i, j}^{-}\right)+\bar{\xi}_{i} \eta_{j}\left(\delta_{i, j}^{-}-c\right),
\end{array}
$$


where $c$ is an arbitrary parameter and $\tilde{c}=1$ or 0 . One can trace the origin of these parameters if one writes down the most general form of the inverse transformations (4.10):

$$
\begin{gathered}
x_{i}=c \sum_{k=-\infty}^{i} \ln b_{k}+(c-1) \sum_{k=i+1}^{\infty} \ln b_{k}, \quad \eta_{i}=-\tilde{c} \sum_{k=-\infty}^{i} \alpha_{k}+(1-\tilde{c}) \sum_{k=i+1}^{\infty} \alpha_{k}, \\
p_{i}=a_{i}, \quad \xi_{i}=e^{-x_{i}} \beta_{i}, \quad \bar{\xi}_{i}=e^{x_{i-1}} \bar{\beta}_{i}, \quad \bar{\eta}_{i}=\bar{\alpha}_{i} .
\end{gathered}
$$

From the Jacobi identities, one can fix the parameter $\tilde{c}$ to be 1 or 0 , while the second parameter $c$ is left arbitrary.

In the canonical basis (4.10), the bosonic Hamiltonians (4.9) become

$$
\begin{aligned}
& H_{1}=-\sum_{i=1}^{n}\left(p_{i}+\xi_{i} \bar{\xi}_{i}\right), \\
& H_{2}=-\sum_{i=1}^{n}\left(\frac{1}{2} p_{i}^{2}+e^{x_{i}-x_{i-1}}+e^{-x_{i}} \bar{\xi}_{i+1}\left(\eta_{i+1}-\eta_{i}\right)+e^{x_{i}} \xi_{i} \bar{\eta}_{i}\right),
\end{aligned}
$$

and they generate, via the first (4.12) and second (4.13) Hamiltonian structures, the following equations [3]:

$$
\begin{gathered}
\partial x_{i}=p_{i}, \quad \partial \bar{\xi}_{i}=e^{x_{i}} \bar{\eta}_{i}, \quad \partial \xi_{i}=e^{-x_{i-1}}\left(\eta_{i}-\eta_{i-1}\right), \\
\partial p_{i}=e^{x_{i+1}-x_{i}}-e^{x_{i}-x_{i-1}}-e^{x_{i}} \xi_{i} \bar{\eta}_{i}-e^{-x_{i}} \bar{\xi}_{i+1}\left(\eta_{i}-\eta_{i+1}\right), \\
\partial \eta_{i}=-e^{x_{i}} \xi_{i}, \quad \partial \bar{\eta}_{i}=e^{-x_{i}} \bar{\xi}_{i+1}-e^{-x_{i-1}} \bar{\xi}_{i} .
\end{gathered}
$$

The parameters $c$ and $\tilde{c}$ do not affect (4.16) via the second Hamiltonian structure (4.13).

We also present the Lagrangian $\mathscr{L}$ and the action $\mathscr{Y}$,

$$
\begin{aligned}
\mathscr{S} & =\int d t \mathscr{L}=\int d t\left[\sum_{j=-\infty}^{\infty} p_{j} \frac{\partial}{\partial t} x_{j}+\xi_{j} \frac{\partial}{\partial t} \bar{\xi}_{j}+\eta_{j} \frac{\partial}{\partial t} \bar{\eta}_{j}-H_{2}\right] \\
& =\int d t \sum_{j=-\infty}^{\infty}\left[-\frac{1}{2}\left(\frac{\partial}{\partial t} x_{j}\right)^{2}+\xi_{j} \frac{\partial}{\partial t} \bar{\xi}_{j}+\eta_{j} \frac{\partial}{\partial t} \bar{\eta}_{j}+e^{x_{i}-x_{i-1}}+e^{-x_{i}} \bar{\xi}_{i+1}\left(\eta_{i+1}-\eta_{i}\right)+e^{x_{i}} \xi_{i} \bar{\eta}_{i}\right] .
\end{aligned}
$$

One can easily verify that the variation of the action $\mathscr{S}$ with respect to the fields $\left\{x_{j}, \xi_{j}, \bar{\xi}_{j}\right.$, $\left.\eta_{j}, \bar{\eta}_{j}\right\}$ produces equations of motion (4.16) for them with reversed sign of the time $(\partial \rightarrow-\partial / \partial t)$ where the momenta $p_{j}$ are replaced by $-(\partial / \partial t) x_{j}$.

\section{Periodic TL hierarchies}

5.1. Periodic $2 \mathrm{D}$ generalized fermionic TL equations. The $n$-periodic $2 \mathrm{D}$ generalized fermionic TL equations (2.3) are characterized by the boundary conditions (2.4d). This system has completely different symmetry properties for odd and even values of the period $n$. From now on we concentrate on the case with even value $n=2 m$ of the period. 
The $2 m$-periodic $2 \mathrm{D}$ generalized fermionic TL equations (2.3) admit the zero-curvature representation

$$
\left[\partial_{1}+L_{2 m}^{-}, \partial_{2}-L_{2 m}^{+}\right]=0
$$

with the $2 m \times 2 m$ matrices $L_{2 m}^{ \pm}$:

$$
\begin{aligned}
& \left(L_{2 m}^{-}\right)_{i, j}=\rho_{i} \delta_{i, j+1}+d_{i} \delta_{i, j+2}+w^{-1}\left(\rho_{1} \delta_{i, 1} \delta_{j, 2 m}+d_{1} \delta_{i, 1} \delta_{j, 2 m-1}+d_{2} \delta_{i, 2} \delta_{j, 2 m}\right), \\
& \left(L_{2 m}^{+}\right)_{i, j}=\delta_{i, j-2}+\gamma_{i} \delta_{i, j-1}+c_{i} \delta_{i, j}+w\left(\delta_{i, 2 m-1} \delta_{j, 1}+\delta_{i, 2 m} \delta_{j, 2}+\gamma_{2 m} \delta_{i, 2 m} \delta_{j, 1}\right), \\
& L_{2 m}^{-}=\left(\begin{array}{ccccccccc}
0 & 0 & 0 & \cdots & 0 & 0 & 0 & \frac{d_{1}}{w} & \frac{\rho_{1}}{w} \\
\rho_{2} & 0 & 0 & \cdots & 0 & 0 & 0 & 0 & \frac{d_{2}}{w} \\
d_{3} & \rho_{3} & 0 & \cdots & 0 & 0 & 0 & 0 & 0 \\
0 & d_{4} & \rho_{4} & \cdots & 0 & 0 & 0 & 0 & 0 \\
& & & \cdots & \cdots & & & & \\
0 & 0 & 0 & \cdots & d_{2 m-2} & \rho_{2 m-2} & 0 & 0 & 0 \\
0 & 0 & 0 & \cdots & 0 & d_{2 m-1} & \rho_{2 m-1} & 0 & 0 \\
0 & 0 & 0 & \cdots & 0 & 0 & d_{2 m} & \rho_{2 m} & 0
\end{array}\right), \\
& L_{2 m}^{+}=\left(\begin{array}{ccccccccc}
c_{1} & \gamma_{1} & 1 & 0 & \cdots & 0 & 0 & 0 & 0 \\
0 & c_{2} & \gamma_{2} & 1 & \cdots & 0 & 0 & 0 & 0 \\
0 & 0 & c_{3} & \gamma_{3} & \cdots & 0 & 0 & 0 & 0 \\
0 & 0 & 0 & c_{4} & \cdots & 0 & 0 & 0 & 0 \\
& & & & \cdots & \cdots & & & \\
0 & 0 & 0 & 0 & \cdots & 0 & c_{2 m-2} & \gamma_{2 m-2} & 1 \\
w & 0 & 0 & 0 & \cdots & 0 & 0 & c_{2 m-1} & \gamma_{2 m-1} \\
w \gamma_{2 m} & w & 0 & 0 & \cdots & 0 & 0 & 0 & c_{2 m}
\end{array}\right) \text {, }
\end{aligned}
$$

where $w$ is the spectral parameter of length dimension $[w]=-m$.

Now, following [14], we give some definitions concerning supermatrices. For any $n \times n$ supermatrix $F$, one can define the Grassmann parities of rows and columns as $p_{\text {row }}(i)$ $\equiv p\left(F_{i, 1}\right)$ and $p_{\mathrm{col}}(j) \equiv p\left(F_{1, j}\right)$, respectively, where $p\left(F_{i, j}\right)$ is the Grassmann parity of the matrix element $F_{i, j}$. For the matrices $L_{2 m}^{ \pm}$, one has $p_{\text {row }}(i)=p_{\text {col }}(i) \equiv p(i)$. Matrix $F$ has certain Grassmann parity if the expression

$$
p(F)=p(i)+p(j)+p\left(F_{i, j}\right)
$$


does not depend on $i$ and $j$. For even $n=2 m$, the matrices $L_{n}^{ \pm}$have Grassmann parity $p\left(L_{2 m}^{ \pm}\right)=0$, while for odd $n=2 m+1$, matrices $L_{2 m+1}^{ \pm}$have no definite parity and, therefore, for odd $n$, the zero-curvature representation (5.1) (as well as the Lax-pair representation in $1 \mathrm{D}$ space) does not make sense.

5.2. Bi-Hamiltonian structure of the periodic $1 \mathrm{D}$ generalized fermionic TL hierarchy. The periodic $1 \mathrm{D}$ generalized fermionic TL equations (2.27) with the $2 m$-periodic boundary conditions (2.4d) can be reproduced via the following Lax-pair representation:

$$
\begin{aligned}
& \partial L_{2 m}=\left[L_{2 m}, L_{2 m}^{-}\right], \quad L_{2 m}=L_{2 m}^{+}+L_{2 m}^{-}, \\
& L_{2 m}=\left(\begin{array}{cccccccccc}
c_{1} & \gamma_{1} & 1 & 0 & \cdots & \cdots & 0 & 0 & \frac{d_{1}}{w} & \frac{\rho_{1}}{w} \\
\rho_{2} & c_{2} & \gamma_{2} & 1 & \cdots & \cdots & 0 & 0 & 0 & \frac{d_{2}}{w} \\
d_{3} & \rho_{3} & c_{3} & \gamma_{3} & \cdots & \cdots & 0 & 0 & 0 & 0 \\
0 & d_{4} & \rho_{4} & c_{4} & \cdots & \cdots & 0 & 0 & 0 & 0 \\
& & & & \cdots & \cdots & \cdots & \cdots & & \\
0 & 0 & 0 & 0 & \cdots & \cdots & c_{2 m-3} & \gamma_{2 m-3} & 1 & 0 \\
0 & 0 & 0 & 0 & \cdots & \cdots & \rho_{2 m-2} & c_{2 m-2} & \gamma_{2 m-2} & 1 \\
w & 0 & 0 & 0 & \cdots & \cdots & d_{2 m-1} & \rho_{2 m-1} & c_{2 m-1} & \gamma_{2 m-1} \\
w \gamma_{2 m} & w & 0 & 0 & \cdots & \cdots & 0 & d_{2 m} & \rho_{2 m} & c_{2 m}
\end{array}\right),
\end{aligned}
$$

where $L_{2 m}^{ \pm}$are defined according to (5.2).

The $2 m$-periodic 1D generalized fermionic TL equations (2.27) possess the bi-Hamiltonian structure which can be easily derived from the first and second Hamiltonian structures (2.28) and (2.29), if one makes changes there according to the substitution

$$
\delta_{i, j+k} \longrightarrow \delta_{i, j+k}+\delta_{i, j-2 m+k}, \quad \delta_{i, j-k} \longrightarrow \delta_{i, j-k}+\delta_{i, j+2 m-k}, \quad k>0,
$$

and changes the sum limits in the Hamiltonians (2.25) as

$$
H_{1}^{2 m}=\sum_{i=1}^{2 m}(-1)^{i} c_{i}, \quad H_{2}^{2 m}=\sum_{i=1}^{2 m}(-1)^{i}\left(\frac{1}{2} c_{i}^{2}+d_{i}+\rho_{i} \gamma_{i-1}\right)
$$

Thus, the first and second Hamiltonians structures are explicitly

$$
\begin{aligned}
& \left\{d_{i}, c_{j}\right\}_{1}=(-1)^{j} d_{i}\left(\delta_{i, j+2}-\delta_{i, j}+\delta_{i, j-2 m+2}\right), \\
& \left\{c_{i}, \rho_{j}\right\}_{1}=(-1)^{j} \rho_{j}\left(\delta_{i, j-1}+\delta_{i, j}+\delta_{i, j+2 m-1}\right), \\
& \left\{\rho_{i}, \rho_{j}\right\}_{1}=(-1)^{j}\left(d_{i}\left(\delta_{i, j+1}+\delta_{i, j-2 m+1}\right)-d_{j}\left(\delta_{i, j-1}+\delta_{i, j+2 m-1}\right)\right), \\
& \left\{\gamma_{i}, \gamma_{j}\right\}_{1}=(-1)^{j}\left(\delta_{i, j+1}-\delta_{i, j-1}+\delta_{i, j-2 m+1}-\delta_{i, j+2 m-1}\right), \\
& \left\{d_{i}, d_{j}\right\}_{2}=(-1)^{j} d_{i} d_{j}\left(\delta_{i, j+2}-\delta_{i, j-2}+\delta_{i, j-2 m+2}-\delta_{i, j+2 m-2}\right),
\end{aligned}
$$




$$
\begin{aligned}
& \left\{d_{i}, c_{j}\right\}_{2}=(-1)^{j} d_{i} c_{j}\left(\delta_{i, j+2}-\delta_{i, j}+\delta_{i, j-2 m+2}\right), \\
& \left\{c_{i}, c_{j}\right\}_{2}=(-1)^{j}\left(d_{i}\left(\delta_{i, j+2}+\delta_{i, j-2 m+2}\right)-d_{j}\left(\delta_{i, j-2}+\delta_{i, j+2 m-2}\right)\right. \\
& \left.\quad-\gamma_{j} \rho_{i}\left(\delta_{i, j+1}+\delta_{i, j-2 m+1}\right)-\gamma_{i} \rho_{j}\left(\delta_{i, j-1}+\delta_{i, j+2 m-1}\right)\right), \\
& \left\{d_{i}, \rho_{j}\right\}_{2}=(-1)^{j} d_{i} \rho_{j}\left(\delta_{i, j+2}+\delta_{i, j-1}+\delta_{i, j-2 m+2}+\delta_{i, j+2 m-1}\right), \\
& \left\{d_{i}, \gamma_{j}\right\}_{2}=(-1)^{j} d_{i} \gamma_{j}\left(\delta_{i, j+2}+\delta_{i, j+1}+\delta_{i, j-2 m+2}+\delta_{i, j-2 m+1}\right), \\
& \left\{c_{i}, \rho_{j}\right\}_{2}=(-1)^{j}\left(c_{i} \rho_{j}\left(\delta_{i, j}+\delta_{i, j-1}+\delta_{i, j+2 m-1}\right)-d_{j} \gamma_{i}\left(\delta_{i, j-2}+\delta_{i, j+2 m-2}\right)\right. \\
& \left.\quad-d_{i} \gamma_{j}\left(\delta_{i, j+1}+\delta_{i, j-2 m+1}\right)\right), \\
& \left\{c_{i}, \gamma_{j}\right\}_{2}=(-1)^{j}\left(\rho_{i} \delta_{i, j+2}+\rho_{j} \delta_{i, j-1}+\delta_{i, j-2 m+2}+\delta_{i, j+2 m-1}\right), \\
& \left\{\rho_{i}, \gamma_{j}\right\}_{2}=(-1)^{j}\left(\rho_{i} \gamma_{j}\left(\delta_{i, j+1}+\delta_{i, j-2 m+1}\right)+d_{i}\left(\delta_{i, j+3}+\delta_{i, j-2 m+3}\right)-d_{j}\left(\delta_{i, j-1}+\delta_{i, j+2 m-1}\right)\right), \\
& \left\{\rho_{i}, \rho_{j}\right\}_{2}=(-1)^{j}\left(\left(\rho_{i} \rho_{j}-d_{j} c_{i}\right)\left(\delta_{i, j-1}+\delta_{i, j+2 m-1}\right)+\left(\rho_{i} \rho_{j}+d_{i} c_{j}\right)\left(\delta_{i, j+1}+\delta_{i, j-2 m+1}\right)\right), \\
& \left\{\gamma_{i}, \gamma_{j}\right\}_{2}=(-1)^{j}\left(c_{i}\left(\delta_{i, j+1}+\delta_{i, j-2 m+1}\right)-c_{j}\left(\delta_{i, j-1}+\delta_{i, j+2 m-1}\right)\right),
\end{aligned}
$$

respectively.

Bosonic integrals of motion of the $2 m$-periodic $1 \mathrm{D}$ generalized TL hierarchy can be derived via the following general formula:

$$
\operatorname{str} L_{2 m}^{k}=\sum_{p=1}^{2 m}(-1)^{p}\left(L_{2 m}^{k}\right)_{p p}=k\left(H_{k}^{2 m}+w I_{k-m}^{2 m}+\frac{\hat{I}_{k+m}^{2 m}}{w}+\delta_{k, 2 m} \frac{\hat{I}_{4 m}^{2 m}}{w^{2}}\right), \quad k=1, \ldots, 2 m .
$$

Here, $H_{k}^{2 m}$ are the bosonic Hamiltonians in (5.6), and $I_{k}^{2 m}$ and $\hat{I}_{k}^{2 m}$ are the additional conserved quantities. We analyzed attentively the quantities $I_{k}^{2 m}$ for the case $m=2,3$ and found that $I_{k}^{2 m}$ can be decomposed into a sum of a few terms which are conserved separately and besides $H_{k}^{2 m}$ contain two more independent integrals of motion of length dimension $k<-1$,

$$
\begin{gathered}
I_{p}^{2 m}=0, \quad \text { if } p \leq 0, \quad I_{1}^{2 m}=H_{1}^{2 m}+\frac{1}{2} S_{3}^{2 m} S_{4}^{2 m}, \\
I_{2}^{2 m}=H_{2}^{2 m}+U_{2}^{2 m}+V_{2}^{2 m}+\frac{1}{2} H_{1}^{2 m} S_{3}^{2 m} S_{4}^{2 m}, \\
\hat{I}_{m+1}^{2 m}=0, \quad \hat{I}_{2 m}^{2 m}=U_{2 m}^{2 m}-V_{2 m}^{2 m},
\end{gathered}
$$

where $U_{k}^{2 m}$ and $V_{k}^{2 m}$ are additional bosonic integrals of motion,

$$
\begin{aligned}
U_{2}^{2 m} & =\sum_{j=1}^{m} \sum_{i=1}^{m} \sum_{k=1}^{m} c_{2 j+1} \gamma_{2 i} \gamma_{2 k-1}+\frac{1}{4} \sum_{j=1}^{2 m} \sum_{i=0}^{m-2} \sum_{k=i+1}^{m-1} \sum_{p=k}^{m-1}(-1)^{j} \gamma_{j} \gamma_{j+2 i+1} \gamma_{j+2 k} \gamma_{j+2 p+1}, \\
V_{2}^{2 m} & =\sum_{j=1}^{2 m} \sum_{i=1}^{m}(-1)^{j}\left(\frac{1}{2} c_{j} c_{j+2 i}-\gamma_{j-1} \rho_{j+2 i}\right)
\end{aligned}
$$




$$
\begin{aligned}
& +\sum_{j=1}^{m}\left(c_{2 j} \sum_{i=0}^{m-2} \sum_{k=i}^{m-2} \gamma_{2 j+2 i+1} \gamma_{2 j+2 k+2}-c_{2 j-1} \sum_{i=0}^{m-1} \sum_{k=i}^{m-1} \gamma_{2 j+2 i-1} \gamma_{2 j+2 k}\right), \\
U_{2 m}^{2 m}= & \prod_{i=1}^{m} d_{2 i}, \quad V_{2 m}^{2 m}=\prod_{i=1}^{m} d_{2 i-1},
\end{aligned}
$$

and $S_{3}^{2 m}$ and $S_{4}^{2 m}$ are fermionic integrals (see (5.11)). Our conjecture is that formulae (5.9) and (5.10) are valid not only for the values $m=2,3$ for which they were actually calculated, but also for an arbitrary value of $m$.

The first fermionic Hamiltonians (2.35) in the $2 m$-periodic case become

$$
S_{1}^{2 m}=\sum_{i=1}^{2 m}(-1)^{i} \rho_{i} g_{i}^{-1}, \quad S_{2}^{2 m}=\sum_{i=1}^{2 m} \rho_{i} g_{i}^{-1}, \quad S_{3}^{2 m}=\sum_{i=1}^{2 m}(-1)^{i} \gamma_{i}, \quad S_{4}^{2 m}=\sum_{i=1}^{2 m} \gamma_{i}
$$

Note that in the periodic case the fields $g_{j}$ are connected with the fields $d_{j}$ via the irreversible relation $d_{j}=g_{j} g_{j-1}$. For the fields $g_{j}$, there are (2.33) and it seems reasonable to consider (2.27), (2.32), and (2.33) as a single joined system of equations. In this case, the system possesses the $N=4$ supersymmetry and has additional bosonic integrals of motion $\hat{V}_{k}^{2 m}$ and $\hat{U}_{k}^{2 m}$ which can be derived using automorphism (2.38),

$$
\begin{aligned}
& \hat{V}_{k}^{2 m}=V_{k}^{2 m}\left(\gamma_{j} \longrightarrow(-1)^{j} \rho_{j+1} g_{j+1}^{-1}, \rho_{j} \longrightarrow(-1)^{j} \gamma_{j-1} g_{j}\right), \\
& \hat{U}_{k}^{2 m}=U_{k}^{2 m}\left(\gamma_{j} \longrightarrow(-1)^{j} \rho_{j+1} g_{j+1}^{-1}, \rho_{j} \longrightarrow(-1)^{j} \gamma_{j-1} g_{j}\right) .
\end{aligned}
$$

We suppose that the Hamiltonians (5.11) are the only independent fermionic integrals of motion which exist for the $2 m$-periodic $1 \mathrm{D}$ generalized fermionic TL equations (2.27). Thus, we have checked that higher fermionic Hamiltonians of length dimensions $-3 / 2$ and $-5 / 2$ in the $2 m$-periodic case for $m=2$ become composite and can be expressed via the fermionic Hamiltonians (5.11) and bosonic integrals of motion as a sum of composite terms.

5.3. The $r$-matrix formalism. There is another approach to reproduce bosonic integrals of motion [7]. We consider the $2 m$-periodic auxiliary linear problem

$$
\begin{aligned}
& \lambda \psi_{j}=\left(L_{2 m}\right)_{i j} \psi_{j} \equiv \rho_{j} \psi_{j-1}+d_{j} \psi_{j-2}+\psi_{j+2}+\gamma_{j} \psi_{j+1}+c_{j} \psi_{j}, \\
& \partial \psi_{j}=\left(L_{2 m}^{-}\right)_{i j} \psi_{j} \equiv \rho_{j} \psi_{j-1}+d_{j} \psi_{j-2},
\end{aligned}
$$

for the wave functions $\psi_{j}$ such that $\psi_{j+2 m}=w \psi_{j}$. One can check that (5.13) and (5.14) are equivalent to the following linear problem:

$$
\Phi_{j+1}=\mathfrak{L}_{j}(\lambda) \Phi_{j}, \quad \partial \Phi_{j}=\mathfrak{U}_{j}(\lambda) \Phi_{j}, \quad \Phi_{j}=\left(\begin{array}{c}
\psi_{j+1} \\
\psi_{j-1} \\
\psi_{j} \\
\psi_{j-2}
\end{array}\right)
$$


where

$$
\begin{aligned}
\mathfrak{L}_{j}(\lambda) & =\left(\begin{array}{cccc}
-\gamma_{j} & -\rho_{j} & \lambda-c_{j} & -d_{j} \\
0 & 0 & 1 & 0 \\
1 & 0 & 0 & 0 \\
0 & 1 & 0 & 0
\end{array}\right), \\
\mathfrak{U}_{j}(\lambda) & =\left(\begin{array}{cccc}
0 & -d_{j+1} & -\rho_{j+1} & 0 \\
1 & c_{j-1}-\lambda & \gamma_{j-1} & 0 \\
0 & -\rho_{j} & 0 & -d_{j} \\
0 & \gamma_{j-2} & 1 & c_{j-2}-\lambda
\end{array}\right),
\end{aligned}
$$

and the 1D generalized fermionic TL equations (2.27) result from the consistency condition

$$
\partial \mathfrak{L}_{j}(\lambda)=\mathfrak{U}_{j+1}(\lambda) \mathfrak{L}_{j}(\lambda)-\mathfrak{L}_{j}(\lambda) \mathfrak{U}_{j}(\lambda)
$$

of the linear system (5.15). We note that the $4 \times 4$ matrix Lax operator $\mathfrak{L}_{j}(\lambda)$ in $(5.16)$ has the fermionic Grassmann parity $p\left(\mathfrak{L}_{j}(\lambda)\right)=1$, according to the definition (5.3). The transformations (2.15) to the new basis $\left\{a_{j}, \bar{a}_{j}, b_{j}, \bar{b}_{j}, \alpha_{j}, \bar{\alpha}_{j}, \beta_{j}, \bar{\beta}_{j}\right\}$ in the space of the functions $\left\{c_{j}, d_{j}, \rho_{j}, \gamma_{j}\right\}$ together with the new definitions

$$
\mathscr{L}_{j}(\lambda) \equiv \mathfrak{L}_{2 j+1}(\lambda) \mathfrak{L}_{2 j}(\lambda), \quad V_{j} \equiv \mathfrak{U}_{2 j}(\lambda), \quad F_{j}=\left(\begin{array}{c}
\phi_{j} \\
\phi_{j-1} \\
\varphi_{j} \\
\varphi_{j-1}
\end{array}\right) \equiv \Phi_{2 j}=\left(\begin{array}{c}
\psi_{2 j+1} \\
\psi_{2 j-1} \\
\psi_{2 j} \\
\psi_{2 j-2}
\end{array}\right)
$$

allow us to rewrite (5.13), (5.14), (5.15), (5.16), and (5.17) in the following equivalent form:

$$
\begin{gathered}
\bar{\beta}_{j} \phi_{j-1}+\bar{b}_{j} \varphi_{j-1}+\varphi_{j+1}-\bar{\alpha}_{j} \phi_{j}+\left(\bar{a}_{j}-\lambda\right) \varphi_{j}=0, \\
\beta_{j} \varphi_{j}+b_{j} \phi_{j-1}+\phi_{j+1}+\alpha_{j+1} \varphi_{j+1}+\left(a_{j}-\lambda\right) \phi_{j}=0, \\
F_{j+1}=\mathscr{L}_{j}(\lambda) F_{j}, \quad \partial F_{j+1}=V_{j}(\lambda) F_{j+1}, \\
\partial \mathscr{L}_{j}(\lambda)=V_{j+1}(\lambda) \mathscr{L}_{j}(\lambda)-\mathscr{L}_{j}(\lambda) V_{j}(\lambda),
\end{gathered}
$$

where

$$
\begin{aligned}
\mathscr{L}_{j}(\lambda) & =\left(\begin{array}{cccc}
\lambda-\alpha_{j+1} \bar{\alpha}_{j}-a_{j} & \alpha_{j+1} \bar{\beta}_{j}-b_{j} & \left(\bar{a}_{j}-\lambda\right) \alpha_{j+1}-\beta_{j} & \bar{b}_{j}-\alpha_{j+1} \\
1 & 0 & 0 & 0 \\
\bar{\alpha}_{j} & -\bar{\beta}_{j} & \lambda-\bar{a}_{j} & -\bar{b}_{j} \\
0 & 0 & 1 & 0
\end{array}\right), \\
V_{j}(\lambda) & =\left(\begin{array}{cccc}
0 & -b_{j} & -\beta_{j} & 0 \\
1 & a_{j-1}-\lambda & \alpha_{j} & 0 \\
0 & -\bar{\beta}_{j} & 0 & -\bar{b}_{j} \\
0 & -\bar{\alpha}_{j-1} & 1 & \bar{a}_{j-1}-\lambda
\end{array}\right) .
\end{aligned}
$$


Now, we introduce a new basis $\left\{p_{j}, \bar{p}_{j}, x_{j}, \bar{x}_{j}, \eta_{j}, \bar{\eta}_{j}, \xi_{j}, \bar{\xi}_{j}\right\}$ in the space of the functions $\left\{a_{j}, \bar{a}_{j}, b_{j}, \bar{b}_{j}, \alpha_{j}, \bar{\alpha}_{j}, \beta_{j}, \bar{\beta}_{j}\right\}$,

$$
\begin{gathered}
a_{i}=p_{i}, \quad b_{i}=e^{x_{i}-x_{i-1}}, \quad \alpha_{i}=\eta_{i-1}-\eta_{i}, \quad \beta_{i}=e^{x_{i}-\bar{x}_{i}} \xi_{i}, \\
\bar{a}_{i}=-\bar{p}_{i}, \quad \bar{b}_{i}=e^{\bar{x}_{i}-\bar{x}_{i-1}}, \quad \bar{\alpha}_{i}=-\bar{\eta}_{i}, \quad \bar{\beta}_{i}=e^{-x_{i-1}+\bar{x}_{i}}\left(\bar{\xi}_{i}-\bar{\xi}_{j-1}\right),
\end{gathered}
$$

such that the first Hamiltonian structure (2.28) becomes canonical,

$$
\left\{x_{i}, p_{j}\right\}_{1}=\delta_{i, j}, \quad\left\{\bar{x}_{i}, \bar{p}_{j}\right\}_{1}=\delta_{i, j}, \quad\left\{\xi_{i}, \bar{\xi}_{j}\right\}_{1}=\delta_{i, j}, \quad\left\{\eta_{i}, \bar{\eta}_{j}\right\}_{1}=\delta_{i, j},
$$

and, after gauge transformation,

$$
F_{j}=\Omega_{j} \widetilde{F}_{j}, \quad \Omega_{j}=\left(\begin{array}{cccc}
1 & 0 & \eta_{j} & 0 \\
0 & -e^{x_{j-1}} & 0 & 0 \\
0 & 0 & 1 & 0 \\
0 & e^{\bar{x}_{j-1}} \bar{\xi}_{j-1} & 0 & -e^{\bar{x}_{j-1}}
\end{array}\right),
$$

the linear problem in (5.20) looks like

$$
\widetilde{F}_{j+1}=\tilde{\mathscr{L}}_{j}(\lambda) \widetilde{F}_{j}
$$

where all matrix entries of the matrix $\tilde{\mathscr{L}}_{j}(\lambda)$ are defined at the same lattice node,

$$
\begin{aligned}
\tilde{\mathscr{L}}_{j}(\lambda) & =\Omega_{j+1}^{-1} \mathscr{L}_{j}(\lambda) \Omega_{j} \\
= & \left(\begin{array}{cccc}
\lambda-p_{j}+\eta_{j} \bar{\eta}_{j} & e^{x_{j}}+e^{\bar{x}_{j}} \bar{\xi}_{j} \eta_{j} & -e^{x_{j}-\bar{x}_{j}} \xi_{j}-\left(p_{j}+\bar{p}_{j}\right) \eta_{j} & -e^{\bar{x}_{j}} \eta_{j} \\
-e^{-x_{j}} & 0 & -e^{-x_{j}} \eta_{j} & 0 \\
-\bar{\eta}_{j} & e^{\bar{x}_{j}} \bar{\xi}_{j} & \lambda+\bar{p}_{j}+\eta_{j} \bar{\eta}_{j} & e^{\bar{x}_{j}} \\
e^{-x_{j}} \bar{\xi}_{j} & 0 & -e^{-\bar{x}_{j}}+e^{-x_{j}} \bar{\xi}_{j} \eta_{j} & 0
\end{array}\right) .
\end{aligned}
$$

Here, we note that the $4 \times 4$ matrix Lax operator $\tilde{\mathscr{L}}_{j}(\lambda)$ in $(5.26)$ has the bosonic Grassmann parity $p\left(\tilde{\mathscr{L}}_{j}(\lambda)\right)=0$, according to the definition (5.3), and

$$
\operatorname{sdet} \tilde{\mathscr{L}}_{j}(\lambda)=1
$$

The matrices $\tilde{\mathscr{L}}_{j}(\lambda)$ obey the $r$-matrix Poisson brackets which are equivalent to the algebra (5.23),

$$
\left\{\tilde{\mathscr{L}}_{i}(\lambda){ }^{\otimes}, \tilde{\mathscr{L}}_{j}(\mu)\right\}=\left[r(\lambda-\mu), \tilde{\mathscr{L}}_{i}(\lambda) \otimes \tilde{\mathscr{L}}_{i}(\mu)\right] \delta_{i, j},
$$

where

$$
r(\lambda-\mu)=\frac{P}{\mu-\lambda}
$$

and

$$
P_{i j ; k l}=(-1)^{p(i) p(j)} \delta_{i, l} \delta_{j, k}
$$


is the permutation matrix. The Grassmann parity function $p(j)=0(1)$ for bosonic (fermionic) rows and columns of a supermatrix, and for the supermatrix $\tilde{\mathscr{L}}_{j}(\lambda)$ in $(5.26)$, we have $p(1)=p(2)=0$ and $p(3)=p(4)=1$. In (5.28), we have used the graded tensor product of two even supermatrices $A$ and $B$ [14]

$$
\left(A^{\otimes} B\right)_{i j ; k l}=(-1)^{p(j)(p(i)+p(k))} A_{i k} B_{j l}
$$

with the properties

$$
\begin{gathered}
A^{\otimes} B=P\left(B^{\otimes} A\right) P, \quad\left\{A^{\otimes} B\right\}=-P\left\{B^{\otimes}, A\right\} P, \\
\left\{A^{\otimes}, B C\right\}=\left\{A^{\otimes}, B\right\}\left(I^{\otimes} C\right)+\left(I^{\otimes} B\right)\left\{A^{\otimes}, C\right\} .
\end{gathered}
$$

As a consequence of (5.28) and (5.32), the monodromy matrix

$$
\widetilde{T}_{m}(\lambda)=\prod_{j=1}^{\stackrel{\curvearrowleft}{m}} \tilde{\mathscr{L}}_{j}(\lambda)
$$

satisfies the following Poisson bracket relation:

$$
\left\{\widetilde{T}_{m}(\lambda){ }^{\otimes} \widetilde{T}_{m}(\mu)\right\}=\left[r(\lambda-\mu), \widetilde{T}_{m}(\lambda)^{\otimes} \widetilde{T}_{m}(\mu)\right] .
$$

It follows from (5.34) that $m$ bosonic integrals of motion are in involution since

$$
\operatorname{str} \widetilde{T}_{m}(\lambda)=\left(\tilde{T}_{m}(\lambda)\right)_{11}+\left(\widetilde{T}_{m}(\lambda)\right)_{22}-\left(\tilde{T}_{m}(\lambda)\right)_{33}-\left(\tilde{T}_{m}(\lambda)\right)_{44}
$$

is a polynomial of degree $m$ in $\lambda$ with integrals of motion as the coefficient functions and

$$
\left\{\operatorname{str} \widetilde{T}_{m}(\lambda), \operatorname{str} \widetilde{T}_{m}(\mu)\right\}=\operatorname{str}\left\{\widetilde{T}_{m}(\lambda)^{\otimes}, \widetilde{T}_{m}(\mu)\right\}=\operatorname{str}\left[r(\lambda-\mu), \widetilde{T}_{m}(\lambda)^{\otimes} \widetilde{T}_{m}(\mu)\right]=0 .
$$

We note that the operator $\mathscr{L}_{j}(\lambda)$ in $(5.18)$ can be represented as a product of two fermionic operators $l_{j}(\lambda)$ and $\bar{l}_{j}(\lambda)$,

$$
\mathscr{L}_{j}(\lambda)=l_{j}(\lambda) \bar{l}_{j}(\lambda), \quad l_{j}(\lambda)=\mathfrak{L}_{2 j+1}(\lambda) W_{j}, \quad \bar{l}_{j}(\lambda)=W_{j}^{-1} \mathfrak{L}_{2 j}(\lambda),
$$

where we have introduced the supermatrix $W_{j}$ which we define as

$$
W_{j}=\left(\begin{array}{cccc}
1 & 0 & 0 & 0 \\
0 & 1 & 0 & 0 \\
0 & 0 & 1 & 0 \\
0 & 0 & 0 & e^{x_{j}-1}
\end{array}\right) .
$$

Then, after the gauge transformation (5.24), the Lax operator $\tilde{L}_{j}(\lambda)$ in (5.26) has the form of the product of two fermionic operators $\tilde{l}_{j}(\lambda)$ and $\tilde{\bar{l}}_{j}(\lambda)$,

$$
\begin{gathered}
\tilde{\mathscr{L}}_{j}(\lambda)=\tilde{l}_{j}(\lambda) \tilde{\bar{l}}_{j}(\lambda), \quad \tilde{l}_{j}(\lambda)=\Omega_{j+1}^{-1} l_{j}(\lambda) \equiv \Omega_{j+1}^{-1} \mathfrak{L}_{2 j+1}(\lambda) W_{j}, \\
\tilde{\bar{l}}_{j}(\lambda)=\bar{l}_{j}(\lambda) \Omega_{j} \equiv W_{j}^{-1} \mathfrak{L}_{2 j}(\lambda) \Omega_{j},
\end{gathered}
$$


and each of them is defined at the same lattice node,

$$
\begin{gathered}
\tilde{l}_{j}(\lambda)=\left(\begin{array}{cccc}
-\eta_{j} & -e^{x_{j}-\bar{x}_{j}} \xi_{j} & \lambda-p_{j} & -e^{x_{j}} \\
0 & 0 & -e^{-x_{j}} & 0 \\
1 & 0 & 0 & 0 \\
0 & -e^{\bar{x}_{j}} & e^{x_{j} \bar{\xi}_{j}} & 0
\end{array}\right), \\
\tilde{\bar{l}}_{j}(\lambda)=\left(\begin{array}{cccc}
-\bar{\eta}_{j} & e^{\bar{x}_{j}} \bar{\xi}_{j} & \lambda+\bar{p}_{j}+\eta_{j} \bar{\eta}_{j} & e^{\bar{x}_{j}} \\
0 & 0 & 1 & 0 \\
1 & 0 & \eta_{j} & 0 \\
0 & -1 & 0 & 0
\end{array}\right) .
\end{gathered}
$$

It would be interesting to establish $r$-matrix Poisson bracket relations (if any) between the fermionic supermatrices $\tilde{l}_{j}(\lambda)$ and $\tilde{\bar{l}}_{j}(\lambda)$.

In order to rewrite the monodromy matrix in terms of the original fields $\left\{d_{j}, c_{j}, \rho_{j}, \gamma_{j}\right\}$, one can perform the inverse gauge transformations

$$
\mathscr{L}_{j}(\lambda)=\Omega_{j+1} \tilde{\mathscr{L}}_{j}(\lambda) \Omega_{j}^{-1}
$$

and define the monodromy matrix

$$
T_{m}(\lambda)=\prod_{j=1}^{\stackrel{\curvearrowleft}{m}} \mathscr{L}_{j}(\lambda)=\Omega_{1}\left(\prod_{j=1}^{\stackrel{\curvearrowleft}{m}} \tilde{\mathscr{L}}_{j}(\lambda)\right) \Omega_{1}^{-1}=\Omega_{1} \widetilde{T}_{m}(\lambda) \Omega_{1}^{-1},
$$

where all the matrix entries are expressed in terms of the fields $\left\{d_{j}, c_{j}, \rho_{j}, \gamma_{j}\right\}$. In (5.42), the periodicity property $\Omega_{m+1}=\Omega_{1}$ of the gauge transformation matrix (5.24) has been used. Relation (5.36) is also true for the monodromy matrix $T(\lambda)$ because of the relation $\operatorname{str} T_{m}^{k}(\lambda)=\operatorname{str} \widetilde{T}_{m}^{k}(\lambda)$. In other words, we have shown that $m$ integrals of the motion being expressed in terms of the original fields $\left\{d_{j}, c_{j}, \rho_{j}, \gamma_{j}\right\}$ are in involution. However, as the decomposition (5.8) shows, for the $2 m$-periodic problem, there are more than $m$ integrals of motion. In order to obtain them, we investigate the decomposition

$$
\operatorname{str} T_{m}^{2}(\lambda)=\sum_{k=0}^{2 m-1} J_{2 m-k}^{2 m} \lambda^{k} .
$$

The first several coefficients $J_{p}^{2 m}$ have the following explicit form:

$$
J_{1}^{2 m}=2 H_{1}^{2 m}-2 S_{3}^{2 m} S_{4}^{2 m}, \quad J_{2}^{2 m}=2 H_{2}^{2 m}-4 V_{2}^{2 m}+8 U_{2}^{2 m}+H_{1}^{2 m} S_{3}^{2 m} S_{4}^{2 m} .
$$

One can see that the additional integrals $V_{2}^{2 m}$ and $U_{2}^{2 m}$ are contained in the coefficient $J_{2}^{2 m}$ in a different combination than in $I_{2}^{2 m}$ in (5.9). This is the way to detect them. We suppose that for integrals of higher length dimensions the situation is the same: there are three independent coefficients $H_{k}^{2 m}, I_{k}^{2 m}$, and $J_{k}^{2 m}$ of length dimension $k$ in decompositions (5.8) and (5.43) and each of them is an independent integral of motion.

Having bosonic and fermionic integrals of motion for the $2 m$-periodic $1 \mathrm{D}$ generalized fermionic TL equations (2.27), it is easy to obtain integrals of motion for the periodic 
$N=4$ (3.4) and $N=2$ (4.3) TL equations. This can be done, respectively, using transformations (2.12) and (2.15) together with the reduction constraints (2.21) and (4.2).

5.4. Spectral curves. The Lax operator $L_{2 m}$ in (5.4) and monodromy matrix $T_{m}$ in (5.42) have the common spectrum

$$
L_{2 m} \psi=\lambda \psi, \quad T_{m} \psi=w \psi
$$

so there exist relations $h(\lambda, w)=0$ and $h^{-1}(\lambda, w)=0$ between them, which are formulated in terms of the characteristic function

$$
h(\lambda, w)=\operatorname{sdet}\left(w-T_{m}(\lambda)\right)
$$

Calculating the superdeterminant and applying the modified Euclidean algorithm [13] to an arbitrary supermatrix $M$ with the parities $p(1)=p(2)=0$ and $p(3)=p(4)=1$, one can find

$$
\operatorname{sdet}(w-M)=\frac{w^{2}+\sigma_{1} w+\sigma_{2}}{w^{2}+\sigma_{3} w+\sigma_{4}}
$$

where all the coefficients $\sigma_{m}$ are expressed in terms of four invariants

$$
\mu_{k}=\operatorname{str} M^{k}=\left(M^{k}\right)_{11}+\left(M^{k}\right)_{22}-\left(M^{k}\right)_{33}-\left(M^{k}\right)_{44}, \quad k=1,2,3,4,
$$

of the matrix $M$,

$$
\begin{gathered}
\sigma_{1}=\sigma_{3}+\epsilon_{1}, \quad \sigma_{3}=\frac{1}{\epsilon_{1}}\left(\frac{\epsilon_{4}}{\epsilon_{3}}+\epsilon_{2}\right), \quad \sigma_{2}=\sigma_{4}+\frac{\epsilon_{4}}{\epsilon_{3}}, \quad \sigma_{4}=\frac{1}{\epsilon_{1}^{2}}\left(\frac{\epsilon_{2} \epsilon_{4}}{\epsilon_{3}}+\epsilon_{3}\right), \\
\epsilon_{1}=-\mu_{1}, \quad \epsilon_{2}=-\frac{1}{2}\left(\mu_{1}^{2}-\mu_{2}\right), \quad \epsilon_{3}=\frac{1}{12} \mu_{1}^{4}+\frac{1}{4} \mu_{2}^{2}-\frac{1}{3} \mu_{1} \mu_{3}, \\
\epsilon_{4}=-\frac{1}{4} \mu_{4} \mu_{1}^{2}+\mu_{2}\left(\frac{1}{24} \mu_{1}^{4}-\frac{1}{8} \mu_{2}^{2}+\frac{1}{3} \mu_{3} \mu_{1}\right) .
\end{gathered}
$$

Now, we adapt formulae (5.47) and (5.49) obtained for an arbitrary matrix $M$ to the case when $M$ is the monodromy matrix $T_{m}(\lambda)$ in (5.42). For the monodromy matrix $T_{m}(\lambda)$, there is a relation

$$
\operatorname{sdet} T_{m}(\lambda)=1
$$

which is a consequence of (5.27) and imposes constraints on the coefficients $\sigma_{k}$. Taking $h(\lambda, w)$ at $w=0$ and using (5.50), one finds $\sigma_{4}^{T_{m}}=\sigma_{2}^{T_{m}}, \epsilon_{4}^{T_{m}}=0$, and

$$
h(\lambda, w)=\frac{w^{2}+\sigma_{1}^{T_{m}} w+\sigma_{4}^{T_{m}}}{w^{2}+\sigma_{3}^{T_{m}} w+\sigma_{4}^{T_{m}}},
$$


where $\sigma_{k}^{T_{m}}=\sigma_{k}\left(\epsilon_{s} \rightarrow \epsilon_{s}^{T_{m}}\right), \epsilon_{4}^{T_{m}}=0$, and $\epsilon_{s}^{T_{m}}=\epsilon_{s}\left(\mu_{k} \rightarrow \operatorname{str} T_{m}^{k}(\lambda)\right), s=1,2,3$. From (5.51), one can obtain two spectral curves as zeros of the numerator and denominator:

$$
\begin{gathered}
\mathscr{P}_{\text {num }}(\lambda, w)=w^{2}+\sigma_{1}^{T_{m}} w+\sigma_{4}^{T_{m}}=0 \\
\mathscr{P}_{\mathrm{den}}(\lambda, w)=w^{2}+\sigma_{3}^{T_{m}} w+\sigma_{4}^{T_{m}}=0
\end{gathered}
$$

The eigenvalues $w$ of the curve $\mathscr{P}_{\text {num }}(\lambda, w)=0$ correspond to the eigenvectors with the even Grassmann parity $p(\psi)=0$, while the eigenvalues of the curve $\mathscr{P}_{\mathrm{den}}(\lambda, w)=0$ correspond to the odd eigenvectors $p(\psi)=1$.

5.5. Reduction: $r$-matrix approach and spectral curves for the periodic $1 \mathrm{D} N=2 \mathrm{TL}$ hierarchy. For completeness, in this section we give a short summary of the $r$-matrix formalism for the periodic $1 \mathrm{D} N=2$ supersymmetric TL equations (4.3). In the case under consideration, the auxiliary linear problem (5.19), being reduced by constraints (2.21) and (4.2), becomes

$$
\begin{gathered}
\bar{\beta}_{j} \phi_{j-1}+\varphi_{j+1}-\bar{\alpha}_{j} \phi_{j}-\left(\frac{\beta_{j} \bar{\beta}_{j}}{b_{j}}+\lambda\right) \varphi_{j}=0, \\
\beta_{j} \varphi_{j}+b_{j} \phi_{j-1}+\phi_{j+1}+\alpha_{j+1} \varphi_{j+1}+\left(a_{j}-\lambda\right) \phi_{j}=0,
\end{gathered}
$$

and it is equivalent to the following linear problem:

$$
\widehat{F}_{j+1}=\hat{\mathscr{L}}_{j}(\lambda) \hat{F}_{j}
$$

where

$$
\hat{\mathscr{L}}_{j}(\lambda)=\left(\begin{array}{ccc}
\lambda-\alpha_{j+1} \bar{\alpha}_{j}-a_{j} & \alpha_{j+1} \bar{\beta}_{j}-b_{j} & -\left(\frac{\beta_{j} \bar{\beta}_{j}}{b_{j}}+\lambda\right) \alpha_{j+1}-\beta_{j} \\
1 & 0 & 0 \\
\bar{\alpha}_{j} & -\bar{\beta}_{j} & \lambda+\frac{\beta_{j} \bar{\beta}_{j}}{b_{j}}
\end{array}\right), \quad \hat{F}_{j}=\left(\begin{array}{c}
\phi_{j} \\
\phi_{j-1} \\
\varphi_{j}
\end{array}\right) .
$$

As concerns the periodic $1 \mathrm{D} N=2 \mathrm{TL}$ equations (4.3), they are equivalent to the lattice zero-curvature representation

$$
\partial \hat{\mathscr{L}}_{j}(\lambda)=\hat{V}_{j+1}(\lambda) \hat{\mathscr{L}}_{j}(\lambda)-\hat{\mathscr{L}}_{j}(\lambda) \hat{V}_{j}(\lambda)
$$

with

$$
\hat{V}_{j}=\left(\begin{array}{ccc}
0 & -b_{j} & -\bar{\beta}_{j} \\
0 & -\lambda-a_{j-1} & \alpha_{j} \\
0 & -\bar{\beta}_{j} & 0
\end{array}\right)
$$




\section{Generalized fermionic discrete Toda hierarchy}

In the canonical basis $\left\{x_{j}, p_{j}, \alpha_{j}, \bar{\alpha}_{j}, \beta_{j}, \bar{\beta}_{j}\right\}$ in (4.10) after the gauge transformation

$$
\widehat{F_{j}}=\widehat{\Omega}_{j} \widehat{\widetilde{F}}_{j}, \quad \widehat{\Omega}_{j}=\left(\begin{array}{ccc}
1 & 0 & \eta_{j} \\
0 & -e^{x_{j-1}} & 0 \\
0 & 0 & 1
\end{array}\right)
$$

(5.54) takes the form

$$
\widehat{\widetilde{F}}_{j+1}=\hat{\widetilde{L}}_{j}(\lambda) \widehat{\widetilde{F}}_{j}
$$

where

$$
\hat{\tilde{L}}_{j}(\lambda)=\hat{\Omega}_{j+1}^{-1} \hat{\mathscr{L}}_{j}(\lambda) \hat{\Omega}_{j}=\left(\begin{array}{ccc}
\lambda+\eta_{j} \bar{\eta}_{j}-p_{j} & e^{x_{j}}+\bar{\xi}_{j} \eta_{j} & -e^{x_{j}} \xi_{j}-\left(p_{j}+\xi_{j} \bar{\xi}_{j}\right) \eta_{j} \\
-e^{-x_{j}} & 0 & -e^{-x_{j}} \eta_{j} \\
-\bar{\eta}_{j} & \bar{\xi}_{j} & \lambda+\xi_{j} \bar{\xi}_{j}+\eta_{j} \bar{\eta}_{j}
\end{array}\right),
$$

and is defined at the same lattice node. The matrices $\hat{\widetilde{L}}_{j}(\lambda)$ have the Grassmann parities $p(1)=p(2)=0$ and $p(3)=1$ and obey the same $r$-matrix Poisson bracket relations (5.28) with the appropriate $r$-matrix.

The equation for eigenvalues

$$
\operatorname{sdet}\left(w-\widehat{T}_{m}(\lambda)\right)=0 \quad \text { or } \quad \infty
$$

of the monodromy matrix

$$
\widehat{T}_{m}(\lambda)=\widehat{\Omega}_{1}^{-1} \hat{\widetilde{T}}_{m}(\lambda) \hat{\Omega}_{1}=\widehat{\Omega}_{1}^{-1}\left(\prod_{j=1}^{\stackrel{m}{m}} \hat{\widetilde{L}}_{j}(\lambda)\right) \hat{\Omega}_{1}=\prod_{j=1}^{\stackrel{m}{m}} \hat{\mathscr{L}}_{j}(\lambda)
$$

is defined by the characteristic function

$$
\hat{h}(\lambda, w)=\frac{w^{2}+\hat{\sigma}_{1} w+\hat{\sigma}_{2}}{w+\hat{\sigma}_{3}}
$$

where all the coefficients are expressed in terms of the invariants of the monodromy matrix:

$$
\begin{gathered}
\hat{\mu}_{k}=\operatorname{str} \hat{T}_{m}^{k}=\left(\hat{T}_{m}^{k}\right)_{11}+\left(\widehat{T}_{m}^{k}\right)_{22}-\left(\widehat{T}_{m}^{k}\right)_{33}, \quad k=1,2,3, \\
\hat{\sigma}_{1}=\hat{\sigma}_{3}+\hat{\epsilon}_{1}, \quad \hat{\sigma}_{2}=\hat{\epsilon}_{1} \hat{\sigma}_{3}+\hat{\epsilon}_{2}, \quad \hat{\sigma}_{3}=\frac{\hat{\epsilon}_{3}}{\hat{\epsilon}_{2}}, \\
\hat{\epsilon}_{1}=-\hat{\mu}_{1}, \quad \hat{\epsilon}_{2}=\frac{1}{2}\left(\hat{\mu}_{1}^{2}-\hat{\mu}_{2}\right), \quad \hat{\epsilon}_{3}=\frac{1}{3} \hat{\mu}_{3}-\frac{1}{2} \hat{\mu}_{2} \hat{\mu}_{1}+\frac{1}{6} \hat{\mu}_{1}^{3} .
\end{gathered}
$$

From the relation $\operatorname{sdet} \widehat{\tilde{\mathscr{L}}}_{j}(\lambda)=\lambda^{-1}$, it follows that $\operatorname{sdet} \widehat{T}_{m}=\lambda^{-m}$ and, consequently, $\hat{\sigma}_{3}=-\lambda^{m} \hat{\sigma}_{2}$. 
5.6. Periodic TL equations in the canonical basis. In the previous subsections, we considered the 1D generalized fermionic TL equations (2.27) with the periodic boundary conditions $(2.4 \mathrm{~d})$. All results obtained there can be easily transferred to the case of the $1 \mathrm{D} N=4$ (3.4) and $N=2$ (4.3) TL equations after transition to the new bases, (2.12) and (2.15), respectively, supplied with the reduction constraints (2.21) and (4.2). In particular, (3.4) and (4.3) with the periodic boundary conditions are $N=4$ and $N=2$ supersymmetric, respectively, and admit a bi-Hamiltonian representation which can be derived if one changes the first and second Hamiltonian structures (3.2), (3.3) and (4.7), (4.8), according to the rule (5.5). In this subsection, we consider (3.4) and (4.3) with the $2 \mathrm{~m}$ periodic and $m$-periodic boundary conditions, respectively, in the canonical basis.

The system (3.4) with the $2 m$-periodic boundary conditions in the canonical basis (3.45) is quite similar to the infinite system (3.4) in the canonical basis considered in Section 3.3. Thus, the constraint

$$
\prod_{k=1}^{2 m}\left(-i g_{k}\right)=1
$$

breaks the $N=4$ supersymmetry to the $N=2$ supersymmetry, and the $2 m$-periodic TL equations (3.4) in the canonical basis have exactly the form (3.49) with the $N=2$ supersymmetric flows (3.55). However, the $2 m$-periodic $N=2$ TL equations (3.49) besides the $N=2$ supersymmetry possess additional four nonlocal fermionic nilpotent symmetries. We present only nonzero flows which generate these symmetries:

$$
\begin{aligned}
& D_{s_{1}} x_{j}=\sum_{k=1}^{2 m}\left(\chi_{k}^{-}-(-1)^{k} \chi_{k}^{+}\right), \quad D_{s_{2}} x_{j}=\sum_{k=1}^{2 m}\left(\chi_{k}^{+}+(-1)^{k} \chi_{k}^{-}\right), \quad D_{s_{3}} \chi_{j}^{+}=\sum_{k=1}^{2 m} p_{k}, \\
& D_{s_{3}} \chi_{j}^{-}=-(-1)^{j} \sum_{k=1}^{2 m} p_{k}, \quad D_{s_{4}} \chi_{j}^{+}=(-1)^{j} \sum_{k=1}^{2 m} p_{k}, \quad D_{s_{4}} \chi_{j}^{-}=\sum_{k=1}^{2 m} p_{k} .
\end{aligned}
$$

These flows anticommute with each other and with the supersymmetric flows in (3.55), except the following nonzero anticommutators:

$$
\left\{\tilde{D}_{1}, D_{s_{2}}\right\}=\partial_{T}, \quad\left\{\tilde{D}_{1}, D_{s_{4}}\right\}=-\partial_{T}, \quad\left\{\tilde{D}_{2}, D_{s_{1}}\right\}=\partial_{T}, \quad\left\{\tilde{D}_{2}, D_{s_{3}}\right\}=-\partial_{T}
$$

where we have introduced the new evolution derivative

$$
\partial_{T} q_{j} \equiv\left\{H_{1}^{2}, q_{j}\right\}_{1}
$$

which gives nontrivial flows only for the fields $x_{j}$,

$$
\partial_{T} x_{j}=-2 \sum_{k=1}^{2 m} p_{k}, \quad \partial_{T} p_{j}=0, \quad \partial_{T} \chi_{j}^{ \pm}=0 .
$$


The $2 m$-periodic $N=2$ TL equations (3.49) can be generated using the Hamiltonian

$$
H_{2}=\sum_{j=1}^{2 m}(-1)^{j}\left(\frac{1}{2} p_{j}^{2}-e^{x_{j}-x_{j-2}}-e^{x_{j}-x_{j-1}}\left(\chi_{j-1}^{-}-(-1)^{j} \chi_{j}^{+}\right)\left(\chi_{j}^{-}+(-1)^{j} \chi_{j-1}^{+}\right)\right)
$$

and the canonical first Hamiltonian structure (3.47). Following the standard procedure, one can derive the Lagrangian $\mathscr{L}$ and the action $\mathscr{T}$,

$$
\begin{aligned}
\mathscr{S}=\int d t \mathscr{L}= & \int d t\left[\sum_{j=1}^{2 m} p_{j} \frac{\partial}{\partial t} x_{j}+\chi_{j}^{-} \frac{\partial}{\partial t} \chi_{j}^{+}-H_{2}\right] \\
=\int d t \sum_{j=1}^{2 m}[ & \frac{1}{2}\left(\frac{\partial}{\partial t} x_{j}\right)^{2}+\chi_{j}^{-} \frac{\partial}{\partial t} \chi_{j}^{+} \\
& \left.+(-1)^{j}\left(e^{x_{j}-x_{j-2}}+e^{x_{j}-x_{j-1}}\left(\chi_{j-1}^{-}-(-1)^{j} \chi_{j}^{+}\right)\left(\chi_{j}^{-}+(-1)^{j} \chi_{j-1}^{+}\right)\right)\right] .
\end{aligned}
$$

The variation of the action $\mathscr{S}$ with respect to the fields $\left\{x_{j}, \chi_{j}^{-}, \chi_{j}^{+}\right\}$produces the equations of motion (3.49) for them with reversed sign of the time $(\partial \rightarrow-\partial / \partial t)$ where the momenta $p_{j}$ are replaced by $(-1)^{j}(\partial / \partial t) x_{j}$.

The situation with the system (4.3) with the $m$-periodic boundary conditions is completely different. We recall that the infinite system (4.3) with the boundary conditions (4.5b) for the fields $b_{j}$ at infinity,

$$
\lim _{j \rightarrow \pm \infty} b_{j}=1
$$

is not supersymmetric because the condition (5.72) spoils the supersymmetric flows (4.4). However, in the $m$-periodic case there is no condition (5.72), and, as we will show, it is possible to build at least the $N=1$ supersymmetric $2 m$-periodic TL equations (4.3) in the canonical basis.

The representation of the fields $b_{j}$ in the $m$-periodic canonical basis (4.10) leads to the following constraint:

$$
\prod_{k=j}^{m} b_{j}=1
$$

and in order to preserve both supersymmetry flows (4.4), one needs to provide simultaneously two additional constraints

$$
\sum_{j=1}^{m}\left(\alpha_{j} \pm \bar{\alpha}_{j}\right)=0
$$


It appears that one can preserve one supersymmetry if one modifies the transition to the canonical basis (4.10) as follows:

$$
\begin{gathered}
a_{i}=p_{i}, \quad b_{i}=e^{x_{i}-x_{i-1}}, \quad \beta_{i}=e^{x_{i}} \xi_{i}, \quad \bar{\beta}_{i}=e^{-x_{i-1}} \bar{\xi}_{i}, \\
\bar{\alpha}_{i}=-\bar{\eta}_{i}, \quad \alpha_{i}=\eta_{i-1}-\eta_{i}+s\left(\bar{\eta}_{i-1}+\bar{\eta}_{i}\right),
\end{gathered}
$$

where $s$ is an arbitrary parameter. In this basis, the $m$-periodic first Hamiltonian structure still has the canonical form (4.12), and using the Hamiltonian

$$
H_{2}=-\sum_{i=1}^{n}\left(\frac{1}{2} p_{i}^{2}+e^{x_{i}-x_{i-1}}+e^{-x_{i}} \bar{\xi}_{i+1}\left(\eta_{i+1}-\eta_{i}-s\left(\bar{\eta}_{i+1}+\bar{\eta}_{i}\right)\right)+e^{x_{i}} \xi_{i} \bar{\eta}_{i}\right),
$$

it generates the following equations:

$$
\begin{gathered}
\partial x_{i}=p_{i}, \quad \partial \bar{\xi}_{i}=e^{x_{i}} \bar{\eta}_{i}, \quad \partial \xi_{i}=-e^{-x_{i-1}}\left(\eta_{i-1}-\eta_{i}+s\left(\bar{\eta}_{i}+\bar{\eta}_{i-1}\right)\right), \\
\partial p_{i}=e^{x_{i+1}-x_{i}}-e^{x_{i}-x_{i-1}}-e^{x_{i}} \xi_{i} \bar{\eta}_{i}-e^{-x_{i}} \bar{\xi}_{i+1}\left(\eta_{i}-\eta_{i+1}+s\left(\bar{\eta}_{i}+\bar{\eta}_{i+1}\right)\right), \\
\partial \eta_{i}=-e^{x_{i}} \xi_{i}+s\left(e^{-x_{i-1}} \bar{\xi}_{i}+e^{-x_{i}} \bar{\xi}_{i+1}\right), \quad \partial \bar{\eta}_{i}=e^{-x_{i}} \bar{\xi}_{i+1}-e^{-x_{i-1}} \bar{\xi}_{i} .
\end{gathered}
$$

One can standardly generate the Lagrangian $\mathscr{L}$ and the action $\mathscr{Y}$,

$$
\begin{aligned}
\mathscr{S}=\int d t \mathscr{L}= & \int d t\left[\sum_{j=1}^{n} p_{j} \frac{\partial}{\partial t} x_{j}+\xi_{j} \frac{\partial}{\partial t} \bar{\xi}_{j}+\eta_{j} \frac{\partial}{\partial t} \bar{\eta}_{j}-H_{2}\right] \\
=\int d t \sum_{j=1}^{n}[ & -\frac{1}{2}\left(\frac{\partial}{\partial t} x_{j}\right)^{2}+\xi_{j} \frac{\partial}{\partial t} \bar{\xi}_{j}+\eta_{j} \frac{\partial}{\partial t} \bar{\eta}_{j} \\
& \left.+e^{x_{i}-x_{i-1}}+e^{-x_{i}} \bar{\xi}_{i+1}\left(\eta_{i+1}-\eta_{i}-s\left(\bar{\eta}_{i+1}+\bar{\eta}_{i}\right)\right)+e^{x_{i}} \xi_{i} \bar{\eta}_{i}\right] .
\end{aligned}
$$

The variation of the action $\mathscr{S}$ with respect to the fields $\left\{x_{j}, \xi_{j}, \bar{\xi}_{j}, \eta_{j}, \bar{\eta}_{j}\right\}$ produces the equations of motion (5.77) for them with reversed sign of the time $(\partial \rightarrow-\partial / \partial t)$ where the momenta $p_{j}$ are replaced by $-(\partial / \partial t) x_{j}$. If, in addition to the momenta, the fields $\eta_{j}$ and $\bar{\eta}_{j}$ are also eliminated from (5.77) by means of the corresponding equations expressing them in terms of the fields $\left\{x_{j}, \xi_{j}, \bar{\xi}_{j}\right\}$ and their derivatives, the remaining equations become

$$
\begin{gathered}
\partial^{2} x_{j}=e^{x_{j+1}-x_{j}}-e^{x_{j}-x_{j-1}}-\xi_{j} \partial \bar{\xi}_{j}+\bar{\xi}_{j+1} \partial \xi_{j+1}, \\
\partial\left(e^{x_{j-1}} \partial \xi_{j}\right)=e^{x_{j-1}} \xi_{j-1}-e^{x_{j}} \xi_{j}, \quad \partial\left(e^{-x_{j}} \partial \bar{\xi}_{j}\right)=e^{-x_{j}} \bar{\xi}_{j+1}-e^{-x_{j-1}} \bar{\xi}_{j} .
\end{gathered}
$$

It is interesting to remark that the dependence of (5.79) on the parameter $s$ completely disappears. 
For every $m$, the system (5.77) possesses the $N=1$ supersymmetry at the unique value $s= \pm 1 / 2$, and the supersymmetry flows $\left(D_{ \pm}^{2}=\mp \partial_{t}\right)$ are

$$
\begin{aligned}
& D_{ \pm} x_{i}=-\eta_{i} \pm \frac{1}{2} \sum_{k=1}^{m-1} \bar{\eta}_{i+k}, \\
& D_{ \pm} p_{i}=e^{x_{i}} \xi_{i} \pm e^{-x_{i}} \bar{\xi}_{i+1}, \\
& D_{ \pm} \xi_{i}= \pm e^{-x_{i-1}}-\xi_{i} \eta_{i} \pm \xi_{i} \bar{\eta}_{i} \pm \frac{1}{2} \sum_{k=1}^{m-1} \xi_{i} \bar{\eta}_{i+k} \\
& D_{ \pm} \bar{\xi}_{i}=e^{x_{i}}+\bar{\xi}_{i} \eta_{i} \mp \bar{\xi}_{i} \bar{\eta}_{i} \mp \frac{1}{2} \sum_{k=1}^{m-1} \bar{\xi}_{i} \bar{\eta}_{i+k} \\
& D_{ \pm} \eta_{i}= \pm p_{i} \pm \frac{1}{2} \sum_{k=1}^{m-1}\left(p_{i+k}+\xi_{i+k} \bar{\xi}_{i+k}\right), \\
& D_{ \pm} \bar{\eta}_{i}=p_{i}+\xi_{i} \bar{\xi}_{i} .
\end{aligned}
$$

To close this section, we only mention that besides the supersymmetry flows in (5.80), the system (5.77) possesses an additional nilpotent symmetry

$$
\begin{array}{cl}
D_{p} x_{i}=\sum_{k=1}^{m} \bar{\eta}_{k}, & D_{p} p_{i}=0, \quad D_{p} \xi_{i}=\xi_{i} \sum_{k=1}^{m} \bar{\eta}_{k}, \\
D_{p} \bar{\xi}_{i}=-\bar{\xi}_{i} \sum_{k=1}^{m} \bar{\eta}_{i}, & D_{p} \eta_{i}=\sum_{k=1}^{m}\left(p_{k}+\xi_{k} \bar{\xi}_{k}\right), \quad D_{p} \bar{\eta}_{i}=0 .
\end{array}
$$

\section{Acknowledgments}

We would like to thank A. P. Isaev, P. P. Kulish, F. Magri, and M. A. Olshanetsky for useful discussions. We would also like to thank P. P. Kulish for giving us reference [13]. This work was partially supported by Russian Foundation for Basic Reseach (RFBR) Grant 03-01-00781, RFBR-DFG Grant 02-02-04002, DFG Grant 436 RUS 113/669, and by the Heisenberg-Landau program.

\section{References}

[1] V. A. Andreev, Odd bases of Lie superalgebras and integrable equations, Teoret. Mat. Fiz. 72 (1987), no. 1, 112-119.

[2] G. Au and B. Spence, Hamiltonian reduction and supersymmetric Toda models, Modern Phys. Lett. A 10 (1995), no. 29, 2157-2168.

[3] L. Bonora and A. Sorin, The $N=2$ supersymmetric Toda lattice hierarchy, Nuclear Phys. B 521 (1998), no. 3, 444-470.

[4] F. Delduc, L. Gallot, and A. Sorin, $N=2$ local and $N=4$ non-local reductions of supersymmetric KP hierarchy in $N=2$ superspace, Nuclear Phys. B 558 (1999), no. 3, 545-572.

[5] V. B. Derjagin, A. N. Leznov, and A. Sorin, The solution of the $N=(0 \mid 2)$ superconformal $f$-Toda lattice, Nuclear Phys. B 527 (1998), no. 3, 643-656.

[6] J. Evans and T. Hollowood, Supersymmetric Toda field theories, Nuclear Phys. B 352 (1991), no. 3, 723-768. 
[7] L. D. Faddeev and L. A. Takhtadjan, Hamiltonian Approach to the Theory of Solitons, SpringerVerlag, Berlin, 1986.

[8] K. Ikeda, A supersymmetric extension of the Toda lattice hierarchy, Lett. Math. Phys. 14 (1987), no. $4,321-328$.

[9] — The super-Toda lattice hierarchy, Publ. Res. Inst. Math. Sci. 25 (1989), no. 5, 829-845.

[10] V. G. Kadyshevsky and A. S. Sorin, Continuum limit of the $N=(1 \mid 1)$ supersymmetric Toda lattice hierarchy, JHEP Proceedings, PrHEP unesp2002, Workshop on Integrable Theories, Solitons and Duality (São Paulo, 2002), http://jhep.sissa.it.

[11] Supersymmetric Toda lattice hierarchies, Integrable Hierarchies and Modern Physical Theories (Chicago, Ill, 2000) (H. Aratyn and A. S. Sorin, eds.), NATO Sci. Ser. II Math. Phys. Chem., vol. 18, Kluwer Academic Publishers, Dordrecht, 2001, pp. 289-316.

[12],$N=(1 \mid 1)$ supersymmetric dispersionless Toda lattice hierarchy, Theoret. and Math. Phys. 132 (2002), no. 2, 1080-1093.

[13] Y. Kobayashi and S. Nagamachi, Characteristic functions and invariants of supermatrices, J. Math. Phys. 31 (1990), no. 11, 2726-2730.

[14] A. G. Kuliš and E. K. Skljanin, Solutions of the Yang-Baxter equation, Zap. Nauchn. Sem. Leningrad. Otdel. Mat. Inst. Steklov. (LOMI) 95 (1980), 129-161.

[15] O. Lechtenfeld and A. Sorin, Fermionic flows and tau function of the $N=(1 \mid 1)$ superconformal Toda lattice hierarchy, Nuclear Phys. B 557 (1999), no. 3, 535-547.

[16] A. N. Leznov and A. S. Sorin, Two-dimensional superintegrable mappings and integrable hierarchies in the (2|2) superspace, Phys. Lett. B 389 (1996), no. 3, 494-502.

[17] The solution of the $N=2$ supersymmetric $f$-Toda chain with fixed ends, Phys. Lett. B 402 (1997), no. 1-2, 87-100.

[18] M. A. Olshanetsky, Supersymmetric two-dimensional Toda lattice, Comm. Math. Phys. 88 (1983), no. 1, 63-76.

V. V. Gribanov: Dzhelepov Laboratory of Nuclear Problems, Joint Institute for Nuclear Research, 141980 Dubna, Moscow Region, Russia

E-mail address: gribanov@thsun1.jinr.ru

V. G. Kadyshevsky: Bogoliubov Laboratory of Theoretical Physics, Joint Institute for Nuclear Research, 141980 Dubna, Moscow Region, Russia

E-mail address: kadyshev@jinr.dubna.su

A. S. Sorin: Bogoliubov Laboratory of Theoretical Physics, Joint Institute for Nuclear Research, 141980 Dubna, Moscow Region, Russia

E-mail address: sorin@thsun1.jinr.ru 


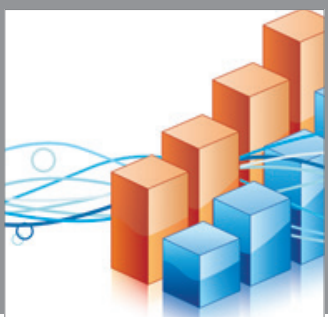

Advances in

Operations Research

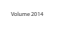

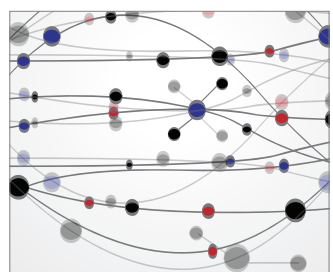

\section{The Scientific} World Journal
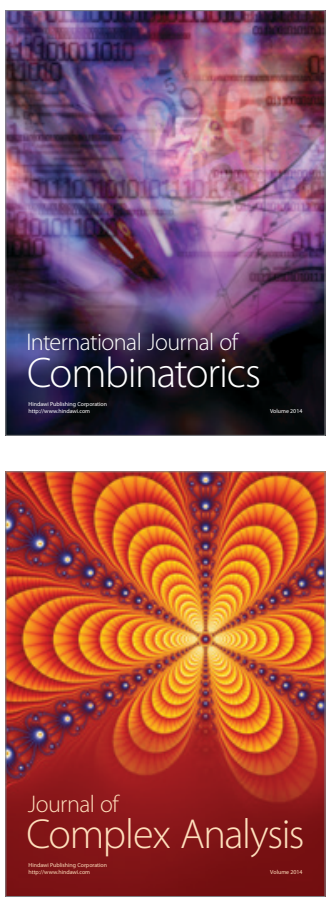

International Journal of

Mathematics and

Mathematical

Sciences
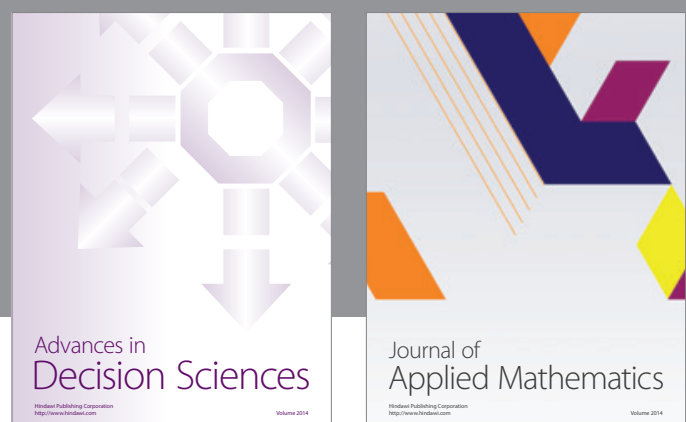

Journal of

Applied Mathematics
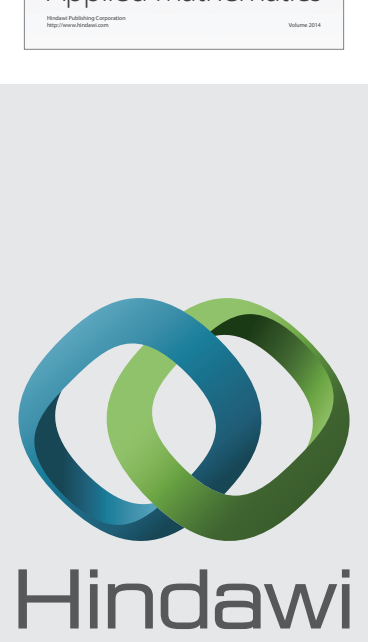

Submit your manuscripts at http://www.hindawi.com
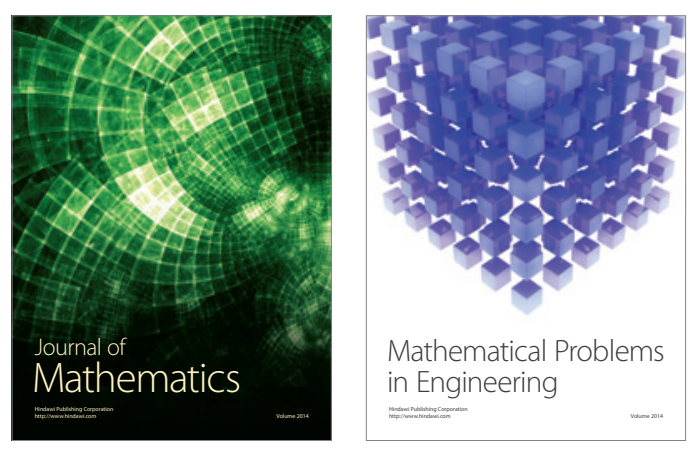

Mathematical Problems in Engineering
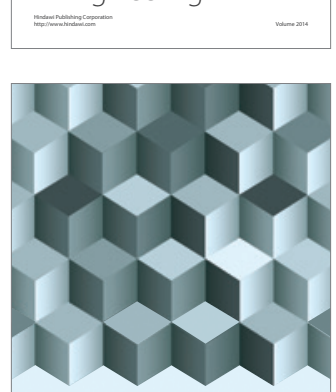

Journal of

Function Spaces
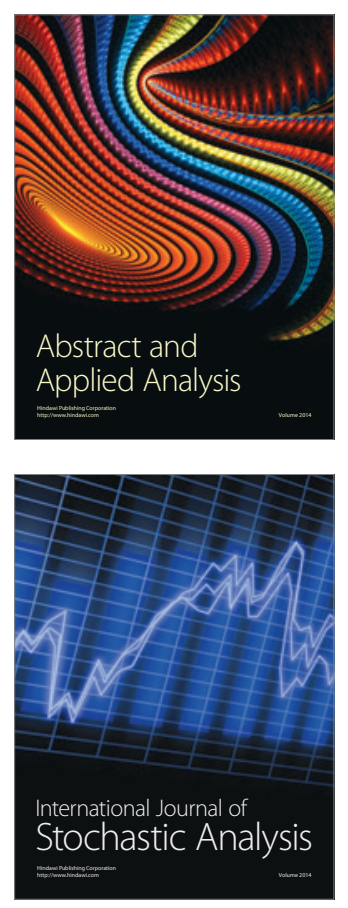

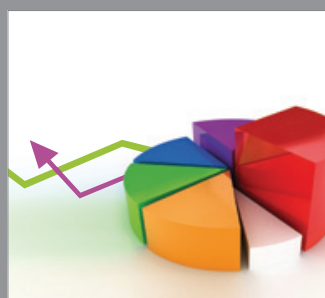

ournal of

Probability and Statistics

Promensencen
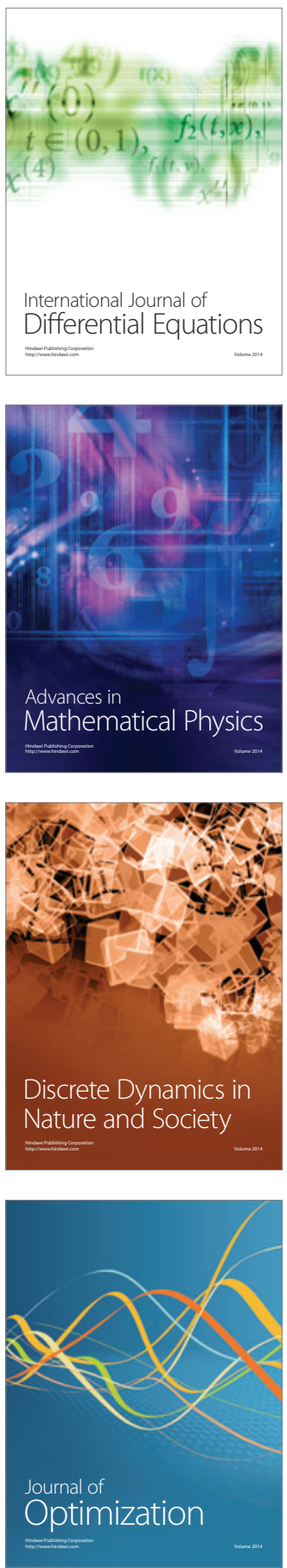